\title{
Predicting Thermal Response of Bridges Using Regression Models Derived from Measurement Histories
}

\author{
Rolands Kromanis*, Prakash Kripakaran \\ College of Engineering, Mathematics and Physical Sciences, University of Exeter, Exeter, UK \\ E-mail: rk296@exeter.ac.uk
}

\begin{abstract}
This study investigates the application of novel computational techniques for structural performance monitoring of bridges that enable quantification of temperature-induced response during the measurement interpretation process. The goal is to support evaluation of bridge response to diurnal and seasonal changes in environmental conditions, which have widely been cited to produce significantly large deformations that exceed even the effects of live loads and damage. This paper proposes a regression-based methodology to generate numerical models, which capture the relationships between temperature distributions and structural response, from distributed measurements collected during a reference period. It compares the performance of various regression algorithms such as multiple linear regression (MLR), robust regression (RR) and support vector regression (SVR) for application within the proposed methodology. The methodology is successfully validated on measurements collected from two structures - a laboratory truss and a concrete footbridge. Results show that the methodology is capable of accurately predicting thermal response and can therefore help with interpreting measurements from continuous bridge monitoring.
\end{abstract}

\section{Introduction}

Rising expenditure on bridge maintenance has led to significant interest in sensing technology and in its potential to lower lifecycle costs of bridge management. Current bridge monitoring systems greatly simplify the collection, storage and transmission of measurements, and such systems are increasingly installed on bridges around the world. Notable examples are the Stonecutters bridge in Hong Kong [1] that is continuously monitored using over 1200 sensors and the St. Anthony Falls bridge in Minnesota, USA [2] that has over three hundred sensors. Monitoring systems on these structures continuously measure a number of bridge parameters related to the structures' response and its environment. The management and interpretation of the collected data, however, poses a great challenge to bridge engineers.

Data interpretation techniques currently employed in practice are often simplistic and tend to be unreliable. For example, the approach typically adopted to analyze measurements from bridge monitoring is to check whether collected measurements exceed pre-defined threshold values and to send email or text notifications to bridge engineers when such situations occur. However, specifying threshold values such that a monitoring system is sensitive to damage while not producing excessive false alarms is seldom possible. The development of robust and reliable strategies for measurement interpretation is therefore increasingly accepted as central to the practical uptake of structural health monitoring (SHM) [3, 4]. Such strategies are also envisioned to be a fundamental element of the future "smart" infrastructures $[3,5,6]$ that are expected to take advantage of recent advances in wireless sensing [7] and energy harvesting technologies [8].

Response measurements (e.g., strains) taken from bridges comprise the effects of several types of loads including vehicular traffic and ambient conditions. Hence an important step in measurement interpretation is characterizing the influences of the individual load components on collected measurements. This research addresses the challenge of accounting for the thermal response in measurements collected during quasi-static monitoring of bridges. Previous long-term monitoring studies have illustrated that daily and seasonal temperature 
variations have a great influence on the structural response of bridges [1,9], and that this influence may even exceed the response to vehicular traffic. Catbas et al. [10] monitored a long-span truss bridge in the USA and observed that the annual peak-to-peak strain differentials for the bridge were ten times higher than the maximum traffic-induced strains. Measurements from long-term monitoring of the Tamar Bridge in the UK showed that thermal variations caused the majority of the observed deformations in the structure (Koo et al., 2012). While this paper is concerned mainly with quasi-static measurements, temperature variations are also known to have noticeable effects on the vibration characteristics of bridges. Cross et al. [11] investigated temperature effects on the dynamic response of Tamar bridge and showed that ambient temperature significantly influences seasonal alterations in the structure's fundamental frequency. Ni et al. [12] and Hua et al. [9] showed that the modal properties such as frequencies are highly correlated with ambient temperatures for the Ting Kau bridge. These studies highlight the importance of temperature effects on long-term structural behaviour. In the context of monitoring, they also emphasize the need for systematic ways of accounting for temperature effects in the measurement interpretation process.

Existing measurement interpretation techniques can be broadly classified into two categories [4] - (1) Model-based (physics-based) methods and (2) Data-driven (non physics-based) methods. Model-based methods rely on behavioural models (e.g., finite element (FE) models) that are derived from the fundamental physics of the structure. Their application to interpreting measurements from continuous structural health monitoring is limited due to their larger computational requirements and the difficulty in generating reliable behaviour models that are representative of the structure $[10,13]$. In contrast to model-based methods, datadriven methods operate directly on measurements and require minimal structural information. They especially show promise for anomaly detection due to their capability for recognizing and tracking patterns in measurement time-series. Data-driven methods use available measurements to first establish baseline criteria for structural behaviour, i.e., to define conditions that identify with normal structural behaviour. For example, measurements collected shortly after a bridge is built or refurbished could help determine baseline conditions. Subsequent measurements are assessed in real-time to detect deviations from these conditions. This research aims to develop computational approaches for evaluating the thermal response of bridges that are suitable for subsequent embedment within data-driven methods for measurement interpretation.

Data-driven methods are typically based on statistical or signal processing techniques such as moving principal component analysis [14] and wavelet transforms [15]. Existing data-driven approaches focus on the analysis of response measurements and ignore distributed temperature measurements. Omenzetter and Brownjohn [16] applied autoregressive integrated moving average models (ARIMA) to analyze strain histories from a full-scale bridge and noted that performance of the models could potentially be improved by including temperature measurements. More recently, Posenato et al. [14] exploited the correlations between response measurements due to seasonal temperature variations for anomaly detection. However, their approach was shown to be sensitive for only high levels of damage. Studies have also treated the effects of temperature variations on structural behaviour as noise superimposed on the traffic-induced response. Laory et al. [17]showed that filtering the effects of diurnal and seasonal temperature changes from response measurements may lead to information loss and hence minimize the chances of detecting anomalies. These results support the development of anomaly detection techniques that explicitly model the relationships between thermal response and distributed temperature measurements. To develop such techniques, systematic approaches for building numerical models that are 
capable of predicting thermal response from distributed temperature measurements are required. This research is a step in that direction.

This paper presents a generic computational approach to evaluate the thermal response of bridges from distributed temperature measurements. The premise of the approach is that a reference set of temperature and response measurements could help train regression-based models for thermal response prediction. This study compares a number of algorithms for model generation including multiple linear regression, support vector regression and artificial neural networks. The goal is to arrive at an approach that leads to robust models that are capable of providing accurate response predictions even when there are noise and outliers in the measurements.

The rest of the paper is organized as follows. The envisioned framework for incorporating thermal effects in the measurement interpretation process is first presented. The proposed approach and the various algorithms that are evaluated for model generation within the approach are then outlined. The two structures - (i) a laboratory truss and (ii) a footbridge at the National Physical Laboratory (NPL), which form the case studies in this research, are described. The performance of the developed approach is studied on measurements from these two structures. Lastly, results are summarized with important conclusions.

\section{Temperature effects in bridges}

Most materials expand or contract under changes in temperature. Therefore bridges and other civil structures continuously deform under temperature distributions that are introduced in them by ambient weather conditions. Temperature distributions across full-scale structures could be very complex depending upon various factors such as their geographic location, their shape and orientation, and the surrounding environment. Temperature gradients in bridges are often non-linear [18] such that they introduce thermal stresses even in bridge girders with simple supports. Potgieter and Gamble [18] showed using measurements from an existing box girder bridge that stresses and forces due to nonlinear temperature distributions are often of magnitudes comparable to those due to live loads. Bridge engineers therefore give significant importance to thermal effects and consequently, bridge designs either incorporate ways of accommodating thermal movements (e.g. expansion joints) or take into account the stresses that could be created by obstruction to thermal movements (e.g. integral bridges). Due to the difficulty in predicting the temperature distributions that could be experienced by as-built structures at the design stage, engineers typically assume linear temperature gradients as indicated by current design codes to evaluate their thermal response. The same approach is, however, not appropriate for interpreting measurements from long-term monitoring since a significant component of response measurements will be due to temperature variations. For example, consider the plot in Figure 1 showing daily variations in bearing displacements for the Cleddau Bridge in Wales. The figure clearly shows that the displacement increases during the day as the temperature rises with dawn and then decreases later in the day with sunset. Moreover, measurements collected over a year (not shown in figure) reveal that the displacements closely follow the seasonal variations in temperatures. 

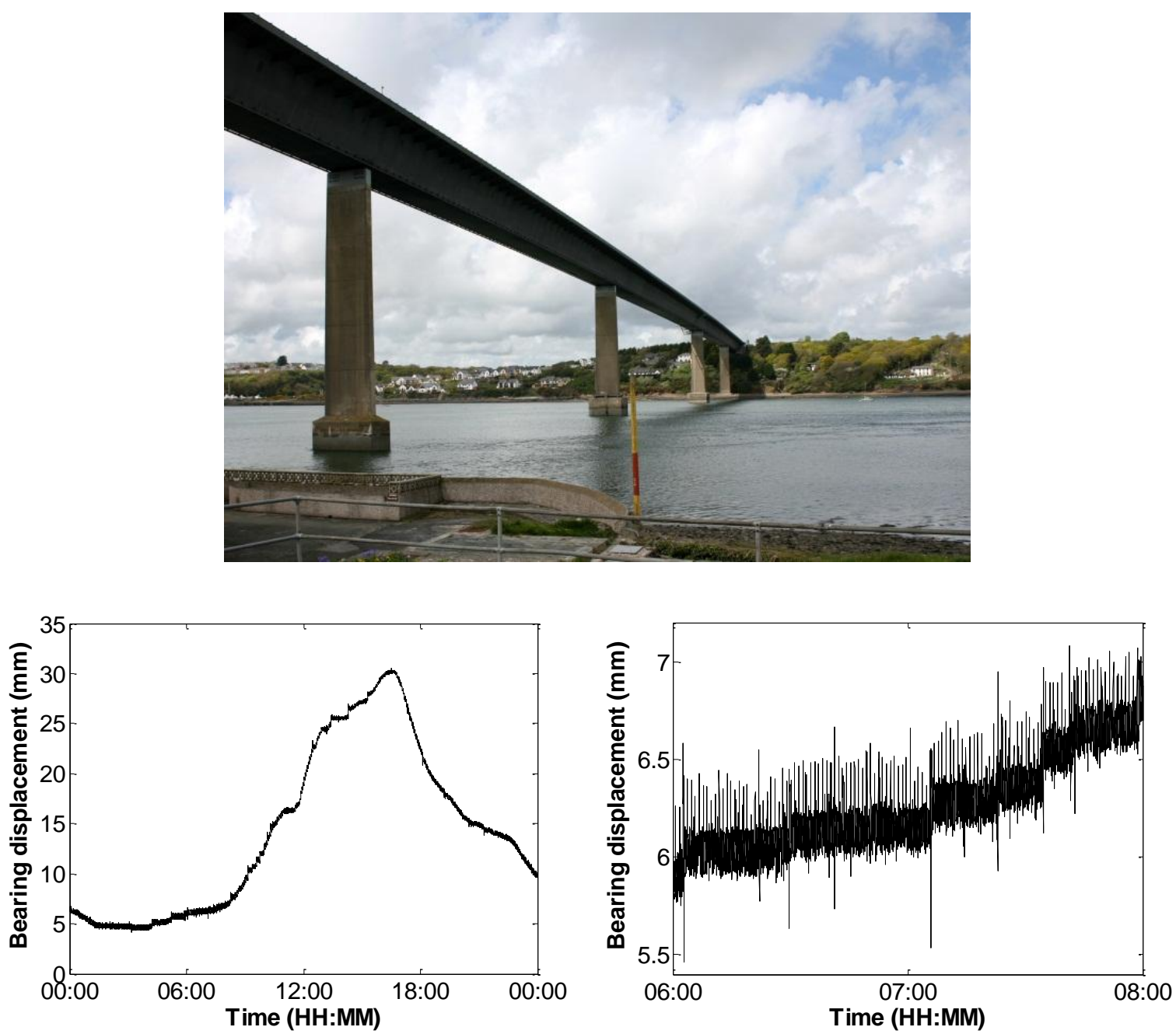

Figure 1: The Cleddau Bridge (top), time series of displacement measurements collected at a bearing over 1 day (bottom left) and a closer look at the measurements taken over 2 hours (bottom right).

(Courtesy: Bill Harvey Associates and Pembrokeshire County Council).

The premise of this study is that temperature effects have to be factored into the measurement interpretation process for the early and reliable detection of abnormal changes in bridge behaviour. This study therefore proposes strategies for predicting and accounting for the thermal response during the measurement interpretation process. The developed strategies will support a bridge management paradigm that is schematically illustrated in Figure 2. A bridge like any structural system exhibits responses that vary according to the applied loads. Loads could be of several types such as vehicular traffic, snow, wind and temperature. A continuous monitoring system measures the integrated structural response (e.g. strains, displacements) of the system to all applied static and dynamic loads. The collected measurements may undergo a preliminary analysis depending on the computing power available on-site and then transmitted to remote servers via the internet or other communication modes. These measurements may be transformed and analyzed through a number of stages of measurement interpretation ranging from initial pre-processing to complex data fusion in order to infer structural performance. This research deals with the development of measurement interpretation strategies to support this part of the bridge management cycle. Results from measurement interpretation may then be presented via suitable interfaces so that engineers are able to plan and prioritize interventions to ensure optimal structural performance. 


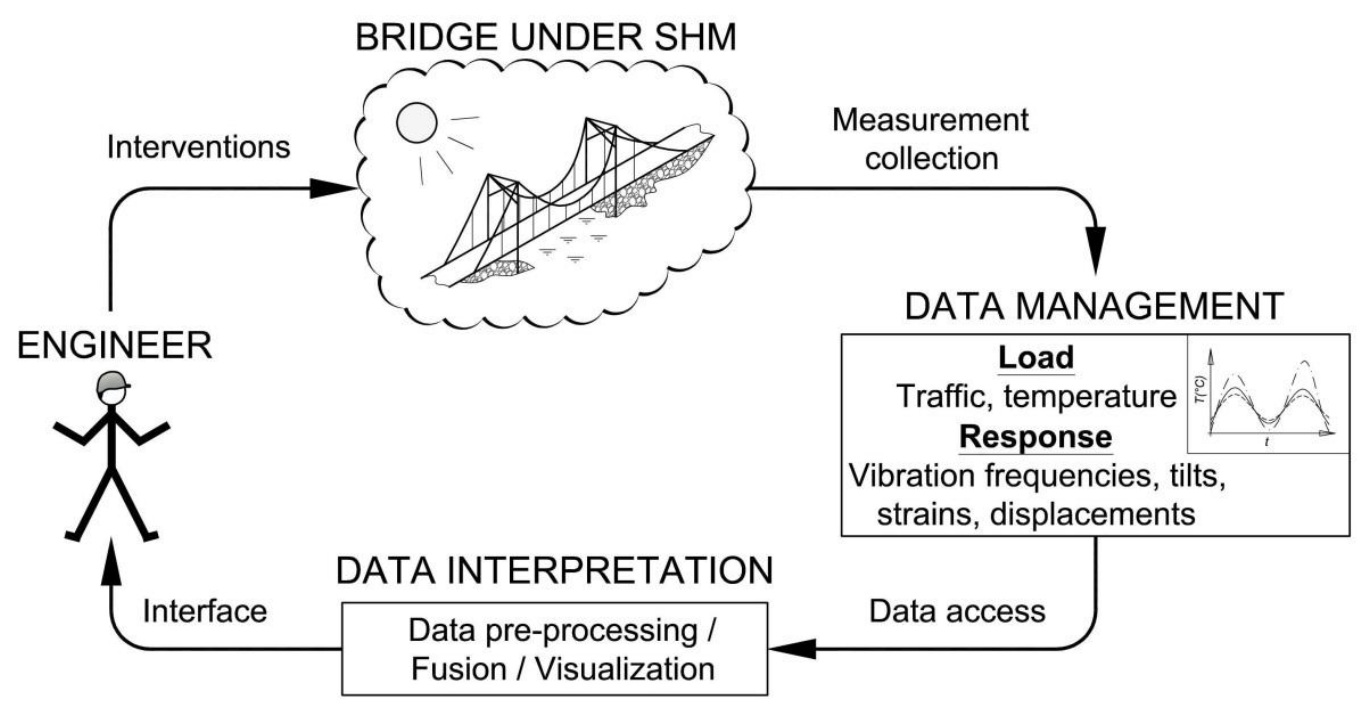

Figure 2: A bridge management paradigm involving SHM.

To be able to detect changes in structural performance from the measured response, it is imperative that we are able to discriminate between the effects of the various loads on response and those due to changes in structural parameters such as stiffness. This study focuses on the development of computational approaches that enable isolating the thermal component of response from measurements. It proposes data-driven strategies for generating statistical models that reliably predict thermal response given a reference set of measurements. It opts for statistical models over physics-based models such as FE models for the following two reasons: (i) ease of transferring the developed approach to a wider range of structures and (ii) suitability for processing voluminous amounts of data collected from continuous monitoring.

The thermal response is directly related to the temperature distribution across the structure. While it is practically impossible to measure temperature at every point on a bridge, measurements from distributed sensing could approximate the temperature distribution in the structure. This research proposes to employ distributed temperature measurements to predict the thermal response of bridges. The methodology is outlined in the form of a flowchart in Figure 3. Temperature and response measurements collected from monitoring are first preprocessed for outliers and noise. Outliers are handled using inter-quartile range analysis. Smoothing techniques are employed to minimize the effects of noise. The dimensionality of the data-sets is then reduced using principal component analysis. This step is performed to generate robust regression models and to minimize the computational expense involved in computing regression models, which typically increases exponentially with increasing numbers of input variables. The pre-processed data is then supplied as input to statistical regression techniques to compute regression models that represent the relationships between temperature distributions and structural response from measurements collected during a reference period. For any chosen regression algorithm, the models are generated iteratively by varying parameter settings until improvements in prediction accuracy are observed to be negligible. The regression model with the best prediction accuracy is then used to predict structural response from collected temperatures, which have been pre-processed for noise and outliers. Residuals between predicted and measured response form time-histories of prediction errors that can later be examined for anomalies. This paper however is focused only on the evaluation of a number of statistical methods for generating regression models starting from simple multiple linear regression to artificial neural networks. From here 
onwards, the approach presented in Figure 3 is referred to as the regression-based thermal response prediction (RBTRP) methodology.

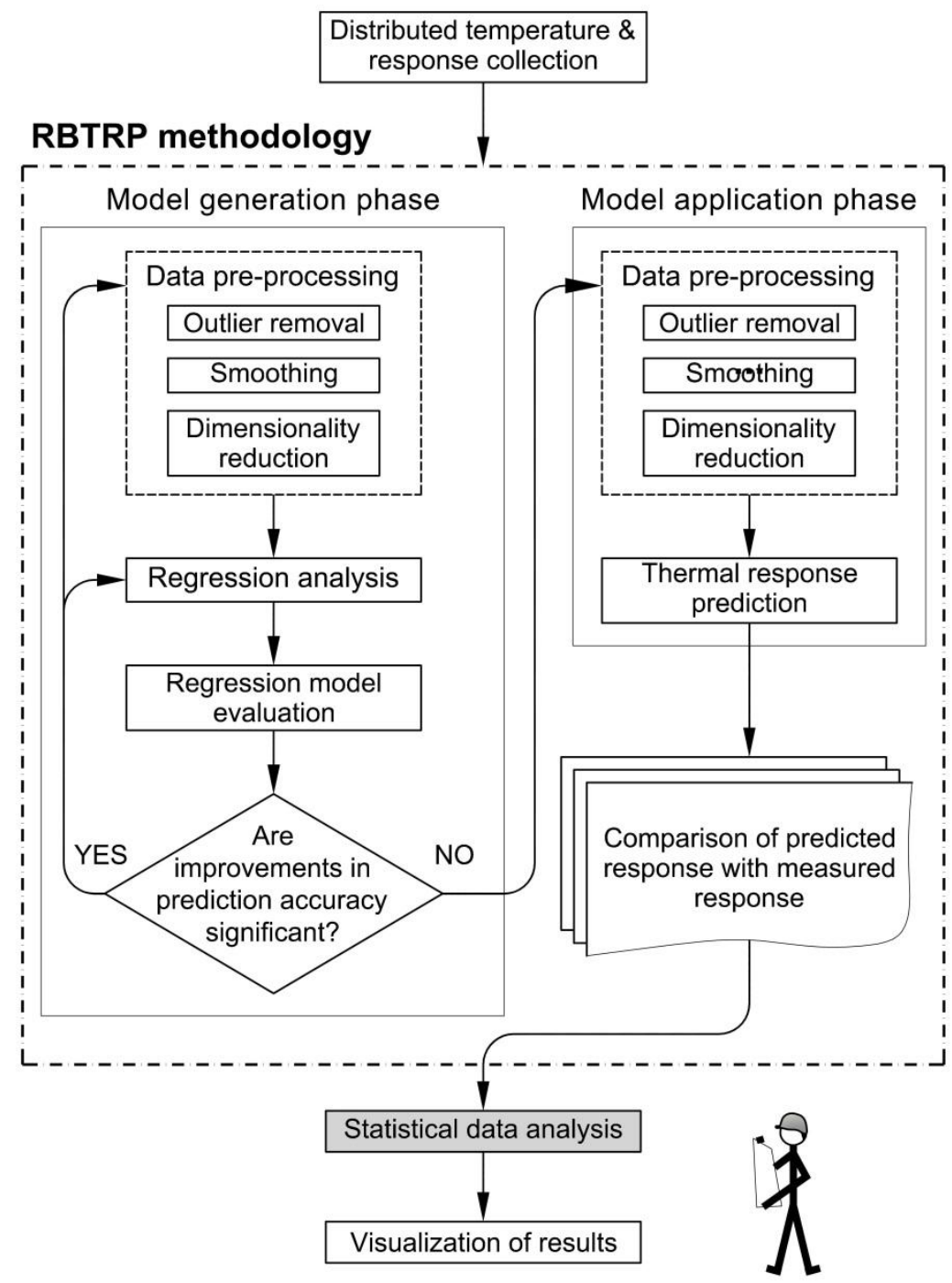

Figure 3: A measurement interpretation approach incorporating temperature effects

\section{Regression algorithms}

Many supervised learning algorithms are potentially capable of generating statistical models that capture the relationship between temperature distributions in a structure and its thermal response. This research investigates application of the following four widely-used supervised learning algorithms for thermal response prediction:

1. Multiple linear regression (MLR) $[19,20]$

2. Robust regression (RR) $[14,21]$

3. Artificial neural networks (ANN) $[22,23]$

4. Support vector regression (SVR) [24, 25]

The above set of algorithms is chosen for the variety they present in terms of computational complexity and due to their previous applications to interpreting measurements from SHM. 


\subsection{Multiple linear regression (MLR)}

MLR is fundamentally an extension of the concept of simple linear regression. In simple regression, available measurements of a dependent variable $(y)$ and an explanatory variable $(x)$ are used to generate a function that can later be used to forecast values for the dependent variable given values for the explanatory variable. However, in many engineering scenarios, multiple explanatory variables may have to be taken together to accurately predict values for a dependent variable [26] and this is accommodated by MLR. The general form of the MLR model (i.e., equation relating the dependent and the explanatory variables) can be expressed as follows:

$$
y_{i}=\beta_{0}+\beta_{1} x_{1 i}+\beta_{2} x_{2 i}+\cdots+\beta_{s} x_{n i}
$$

$y_{i}$ is the predicted value of the dependent variable $y$ for the $i^{\text {th }}$ time step; $n$ is the number of explanatory variables; $x_{1 i}, x_{2 i} \ldots x_{n i}$ are the values for the explanatory variables $\left(x_{1}, x_{2}, \ldots x_{n}\right)$ at the $i^{\text {th }}$ time step; $\beta_{0}$ is the intercept and $\left(\beta_{1}, \beta_{2} \ldots \beta_{n}\right)$ are regression weights. The values for $\beta_{i}$ are computed to minimize an error function based on least-squares estimates. In this study, $y$ represents a thermal response parameter such as displacement or strain at a specific location. $\left(x_{1}, x_{2}, \ldots x_{n}\right)$ represent parameters derived from the temperature measurements recorded by available sensors.

\subsection{Robust regression (RR)}

Regression techniques typically use a least-squares fitting criterion to identify values for the parameters in the regression model. However, this criterion is known to be sensitive to the presence of outliers in the datasets and may therefore lead to unreliable models [27]. RR mitigates this problem by employing a fitting criterion that eliminates outlier-induced bias in the regression model. This criterion is often implemented as a weighted least-squares function where weights are assigned to individual data-sets. The values for the weights are determined in an iterative manner. Initially identical values are assigned to all of them. In subsequent iterations, new values are chosen for the weights based on the errors in model predictions such that higher values are given to data-sets that produce more accurate predictions. This process is terminated when there are minimal changes to the values of the weights between iterations.

\subsection{Artificial neural networks (ANN)}

ANNs [28], which are inspired by biological neural systems, are a powerful way of producing nonlinear regression models between a number of input and output parameters using large numbers of training sets. A neural network consists of neurons that are inter-connected in various layers. The connections between the neurons have weights associated with them and these are calibrated during training to capture the actual relationship between the input and output parameters. In this research, ANNs are simulated using MATLAB's [29] neural network toolbox. A key step is the selection of an appropriate architecture of the network that maximizes its efficiency, i.e., use low computational resources while achieving high prediction accuracy [30]. Similar research in SHM on the application of ANNs for data interpretation recommend using a hidden layer composed of between 3 and 30 neurons [31, 32]. The number of neurons for the hidden layer can also be defined from a general rule-ofthumb $N^{1 / 3}$, where $N$ is the number of input points [33]. This study uses a multi-layer feedforward neural network that implements the back-propagation rule. It has one hidden layer and one output layer of a linear neuron. The optimal number of neurons for the hidden layer is found through a trial and error approach that gradually increases the number of neurons while 
evaluating the performance of the ANN on both training and test sets. A hidden layer of 10 neurons is observed to produce consistently good results. The input parameters to the ANN are derived from distributed temperature measurements. The output parameters are response values (e.g. strains, tilts) at specific locations on the structure.

\subsection{Support vector regression (SVR)}

Support vector machines (SVM) are a class of powerful supervised learning algorithms that are widely used for statistical learning and pattern recognition. The origins of statistical learning theory including the basic principles of SVM can be tracked to the 1960s when it was first introduced by Vladimir Vapnik [34]. These concepts have since been further developed and successfully applied to a range of subject domains such as finance, computer networks and fault detection [34, 35]. This research employs SVM in its regression mode, which is often referred to as support vector regression (SVR). In brief, the main steps in SVR are (i) generation of a vector mapping from the input space to a feature space using kernels, (ii) construction of a separating hyperplane in the feature space and (iii) introduction and modification of a loss function. Further details on the SVM approach are provided in Kromanis and Kripakaran [25].

\section{Case studies}

This research investigates the application of RBTRP methodology on measurements collected from two structures: a laboratory truss and the National Physical Laboratory (NPL) footbridge. The former is used to develop and validate the proposed ideas. The latter is used primarily to highlight the scalability of the developed methodology.

\subsection{Laboratory truss}

An aluminium truss (Figure 4) that is representative of trusses commonly used in short span railway bridges has been fabricated at Exeter as a laboratory-scale structure for this research. The truss is composed of aluminium elements inter-connected with six high strength steel bolts at the joints. Two channel sections, each of size $51 \mathrm{~mm} \times 25 \mathrm{~mm} \times 3 \mathrm{~mm}$, arranged in the shape of an "I", form the top and bottom chords as well as the two end-diagonal elements that connect the top and bottom chords. The other elements are made up of flat bars of size $32 \mathrm{~mm} \times 3 \mathrm{~mm}$. The truss is connected to concrete blocks which are fully fixed to the steel floor, thus, preventing both ends of the truss from any horizontal translation.

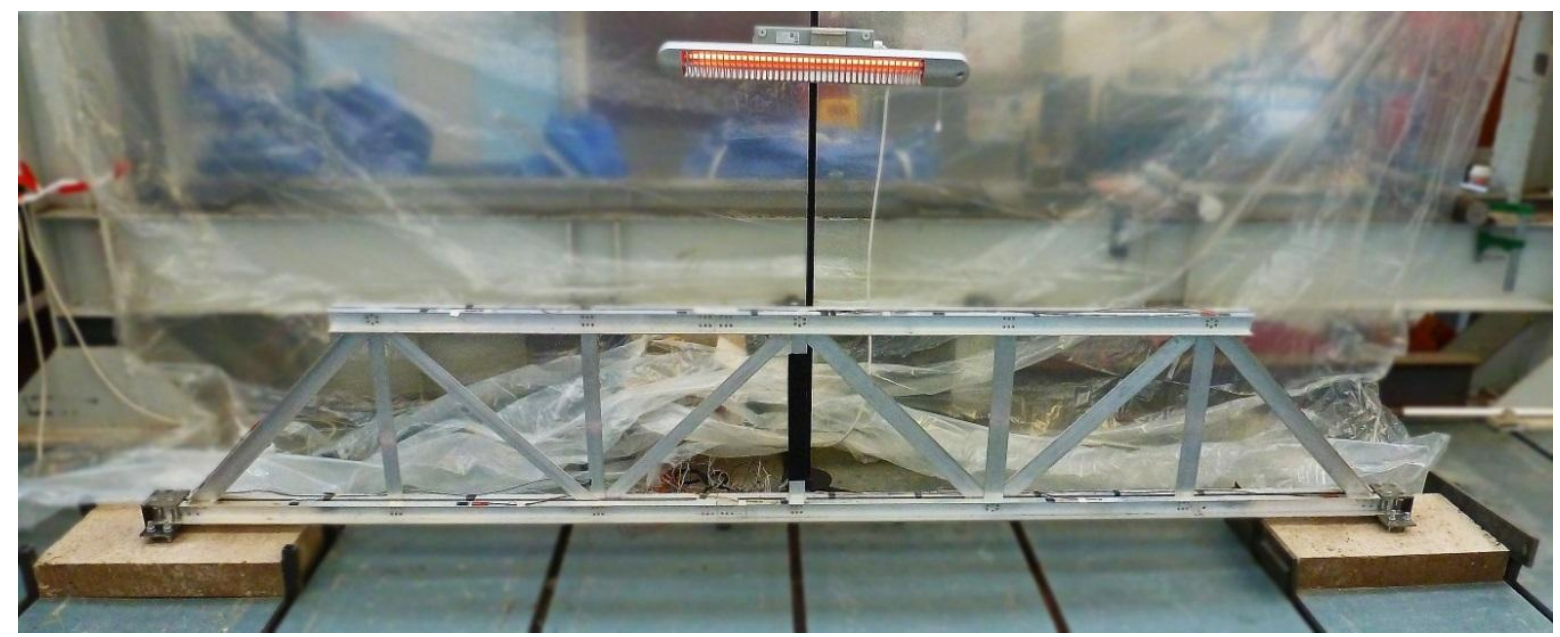

Figure 4: Photograph showing the truss and the infra-red heater 
An infrared heater, with a maximum output of $2 \mathrm{~kW}$, is installed close to the truss. The vertical and horizontal distances between the heater and the middle of the top-chord of the truss are $0.5 \mathrm{~m}$ and $0.15 \mathrm{~m}$ respectively (see Figure 5). The heater is connected to a timer that switches it on automatically for a period of one hour every three hours to approximately emulate diurnal temperature variations; in this experiment, one simulated day lasts 3 hours. As shown in Figure 5, strain and temperature sensors are installed at a number of locations on the truss. Strain sensors are simple resistance-based strain gauges. Material temperature is monitored using K-type thermocouples and thermistors; both provide precise temperature measurements. These sensors are connected to a data-logger unit that is programmed to record measurements every 5 minutes.

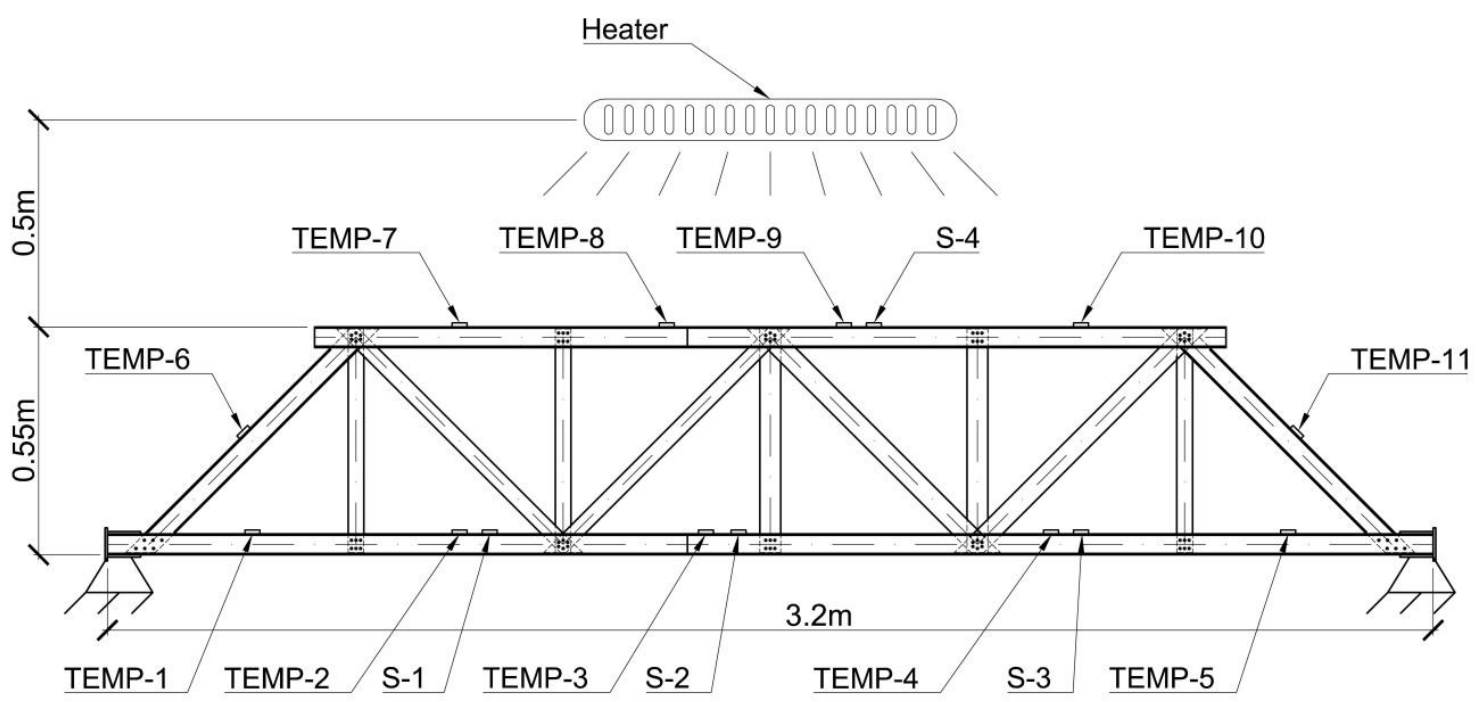

Figure 5: A sketch of the laboratory structure showing its dimensions and the locations of installed thermocouples (TEMP- $i(i=1,2 \ldots 11))$ and strain gauges $(\mathrm{S}-i(i=1,2 \ldots 4))$.

This paper employs measurements collected over a period of 16 days. In total, 4590 measurements have been taken with each of the 15 sensors. Changes in the material temperature and strains are influenced by variations in the ambient temperature in the laboratory and by the radiation from the heater (see Figure 5). The range of temperature and strain values recorded during the monitoring period is provided in Tables 1 and 2. As would be expected, the ranges of temperature and strain measurements are largest at the sensors closer to the heater (Tables 1 and 2 - shaded columns). Data in Tables 1 and 2 confirm that the experiments produce temperature gradients in the truss and therefore result in thermal deformations. Strains measured at sensor S-4 and ambient temperature are plotted in Figure 6. The short, cyclic variations of the strains in the figure are due to the operation of the infra-red heat lamps. The variations in the moving average of the strain time-series are induced by the daily variations in ambient temperature.

Table 1: Maximum and minimum temperatures from the laboratory truss

\begin{tabular}{lccccccccccc}
\hline & \multicolumn{10}{c}{ Temperature sensor (TEMP-i) } \\
\hline$i$ & 1 & 2 & 3 & 4 & 5 & 6 & 7 & 8 & 9 & 10 & 11 \\
\hline $\operatorname{Max}\left({ }^{\circ} \mathrm{C}\right)$ & 20.7 & 21.8 & 24.5 & 21.3 & 21.0 & 21.0 & 22.0 & 30.3 & 28.1 & 20.9 & 21.7 \\
$\operatorname{Min}\left({ }^{\circ} \mathrm{C}\right)$ & 14.9 & 15.2 & 15.2 & 15.2 & 16.0 & 15.6 & 15.9 & 16.1 & 16.1 & 16.0 & 16.2 \\
\hline Range $\left({ }^{\circ} \mathrm{C}\right)$ & 5.8 & 6.5 & 9.3 & 6.1 & 5.0 & 5.4 & 6.2 & 14.2 & 12.0 & 4.8 & 5.5 \\
\hline
\end{tabular}


Table 2: Maximum and minimum strain measurements from the laboratory truss

\begin{tabular}{lcccc}
\hline & S-1 & S-2 & S-3 & S-4 \\
\hline Maximum strain $\left(\times 10^{-6}\right)$ & 20.1 & 37.1 & 49.6 & 97.1 \\
Minimum strain $\left(\times 10^{-6}\right)$ & -24.3 & -14.5 & 7.9 & -37.1 \\
\hline Range $\left(\times 10^{-6}\right)$ & 44.5 & 51.6 & 41.7 & 134.1 \\
\hline
\end{tabular}
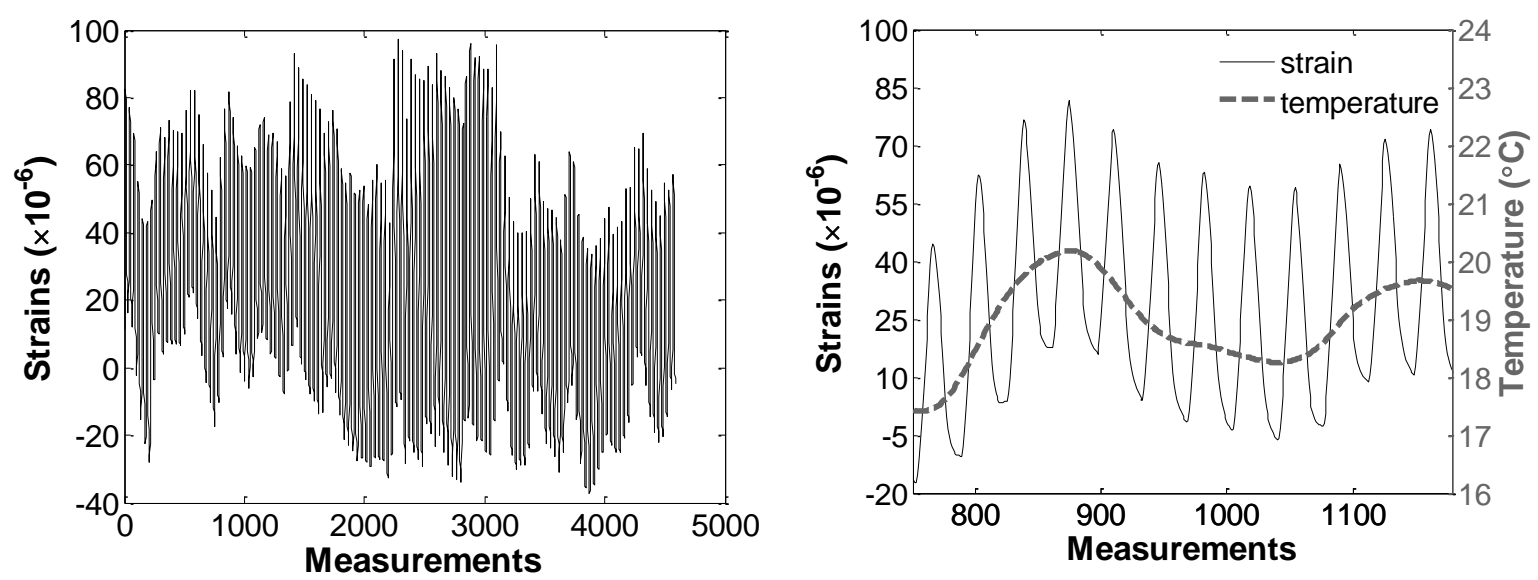

Figure 6: Strain measurements from S-4 for the monitoring period (left) and a zoomed-in view for $1 / 10^{\text {th }}$ of the monitoring period.

\subsection{The National Physical Laboratory (NPL) footbridge project}

A footbridge (Figure 7), which served as a pedestrian passage across the entrance to the National Physics Laboratory (NPL) for more than four decades, was removed from its original location and relocated for monitoring purposes without damaging its integrity and keeping the original boundary conditions. The main span of the bridge is $10.5 \mathrm{~m}$. The bridge has cantilevers at either end ranging between 4.5 and 4.75 meters; these dimensions describe the bridge at the beginning of the monitoring phase. The bridge has been continuously monitored since 2009 with a number of sensors such as optical fibre Bragg grating (FBG) sensors, acoustic emission sensors and wireless accelerometers. Many surveying-based monitoring techniques including digital image correlation and laser scan have also been carried out. The footbridge has also been a test-bed for studying important structural issues such as performance of retrofits and bridge behaviour under damage. 

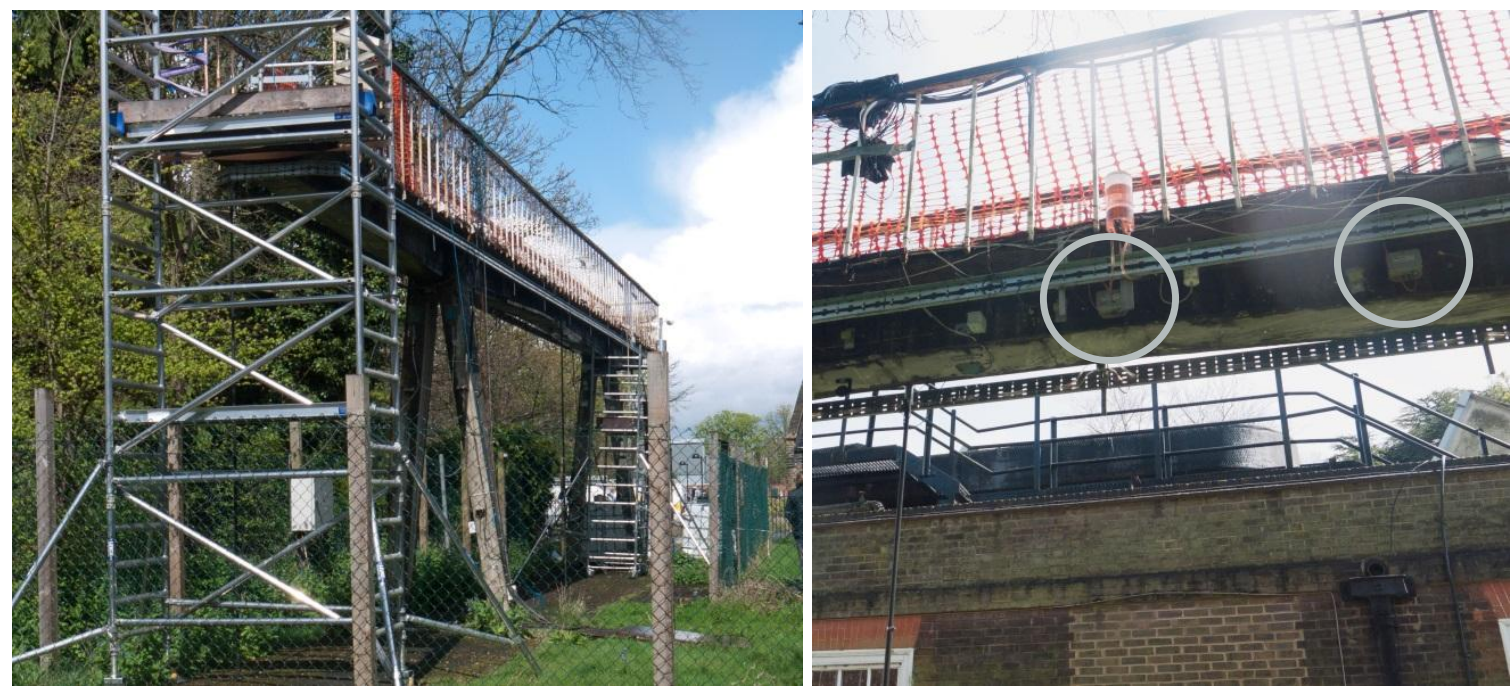

Figure 7: Front view of the NPL footbridge (left) and back view of mid-section of the footbridge (right) and a tilt-meter (circled).

This study draws upon tilt and temperature measurements from 4 tilt sensors and 10 vibrating wire strain gauges with temperature sensors. Since this paper uses only the temperature measurements from the vibrating wire strain gauges, we here onwards refer to these sensors as temperature sensors. Technical details of the sensors are provided in Table 3 and their locations are illustrated in Figure 8.

Table 3: Technical specifications of the tilt and temperature sensors employed in the monitoring of the NPL footbridge

\begin{tabular}{|c|c|c|}
\hline Sensor & Specifications & \\
\hline $\begin{array}{l}\text { Electrolevel surface mount } \\
\text { tilt meter }\end{array}$ & $\begin{array}{l}\text { Range: } \\
\text { Resolution: }\end{array}$ & $\begin{array}{l} \pm 45 \text { Arc Minutes }( \pm 13 \mathrm{~mm} / \mathrm{m}) \\
5.2 \times 10^{-3} \mathrm{~mm} / \mathrm{m}\end{array}$ \\
\hline $\begin{array}{l}\text { Vibrating wire arc- } \\
\text { weldable strain gauge* } \\
\text { with temperature sensor }\end{array}$ & $\begin{array}{l}\text { Temperature range: } \\
\text { Thermistor resolution: }\end{array}$ & $\begin{array}{l}-20 \text { to } 80^{\circ} \mathrm{C} \\
\pm 0.01^{\circ} \mathrm{C}\end{array}$ \\
\hline
\end{tabular}




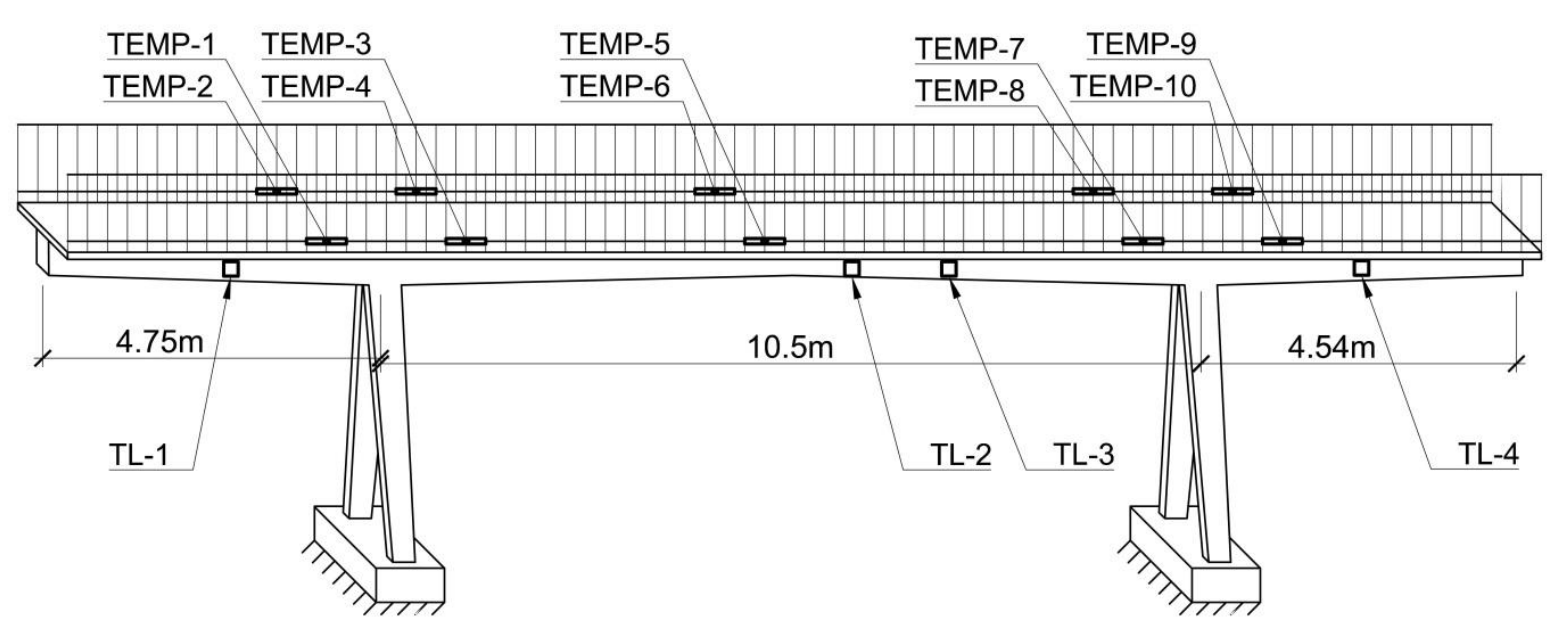

Figure 8: Location of sensors and principal dimensions of the NPL footbridge. TEMP - temperature sensor; TL - tilt sensors. TL-2 and TL-3 are shown in Figure 7 (right (circled)).

The frequency of measurement collection has varied between 1 and 60 measurements per hour over the six months of monitoring due to various circumstances related to the work carried out on the structure. Monitoring was also briefly suspended for a few days. However, for ease of data management, this study considers only the measurements taken at 5-minute intervals and ignores the rest. This results in 30,448 measurements for each sensor.

In contrast to the laboratory structure, the NPL footbridge is exposed to naturally varying environmental conditions and monitored for a longer period. Hence measurements reflect the effects of diurnal and seasonal variations in temperatures. Capturing the full range of tilt and temperature variations requires measurements taken over a six-month period, i.e., from peak winter to peak summer. However, only measurements taken during the first 6 months are useful to validate the proposed methodology since damage and other experimental work affecting the behaviour of the structure is known to be undertaken after this initial period. We overcome this issue by extrapolating the data-set originally collected over duration of six months to two years. This is done by taking advantage of the high frequency of measurement collection as follows. Two data sets (D1 and D2) of equal size are first generated from the original dataset $(D)$. Odd-numbered items in the original time-series $D$ of measurements form $D 1$. The even-numbered items are mirrored about the 6-month timeline and this forms D2. D2 is appended to $D 1$ hence creating a new data-set $E$, which emulates approximately one full season, i.e., 1 year. The process is repeated for $E$ by mirroring half its data-set about the 1year timeline and, as a result, a data-set having duration of two years is created. In this manner, temperature and tilt measurements collected from all the sensors over 6 months are extrapolated to two years. These data-sets are used to validate the RBTRP methodology.

Measurements from the tilt meters TL-1, TL-2, TL-3 and TL-4 are plotted in Figures 9 and 10 and the maximal and minimal tilt values that they recorded are listed in Table 4. TL-1 and TL-4 are located on the two cantilevered ends of the footbridge; thus, the tilt measurements from the two sensors have similar magnitudes but in opposite directions (see Figure 9). In contrast, measurements from TL-2 and TL-3, which are located just to the right of mid-span of the footbridge, have similar patterns (see Figure 10). 

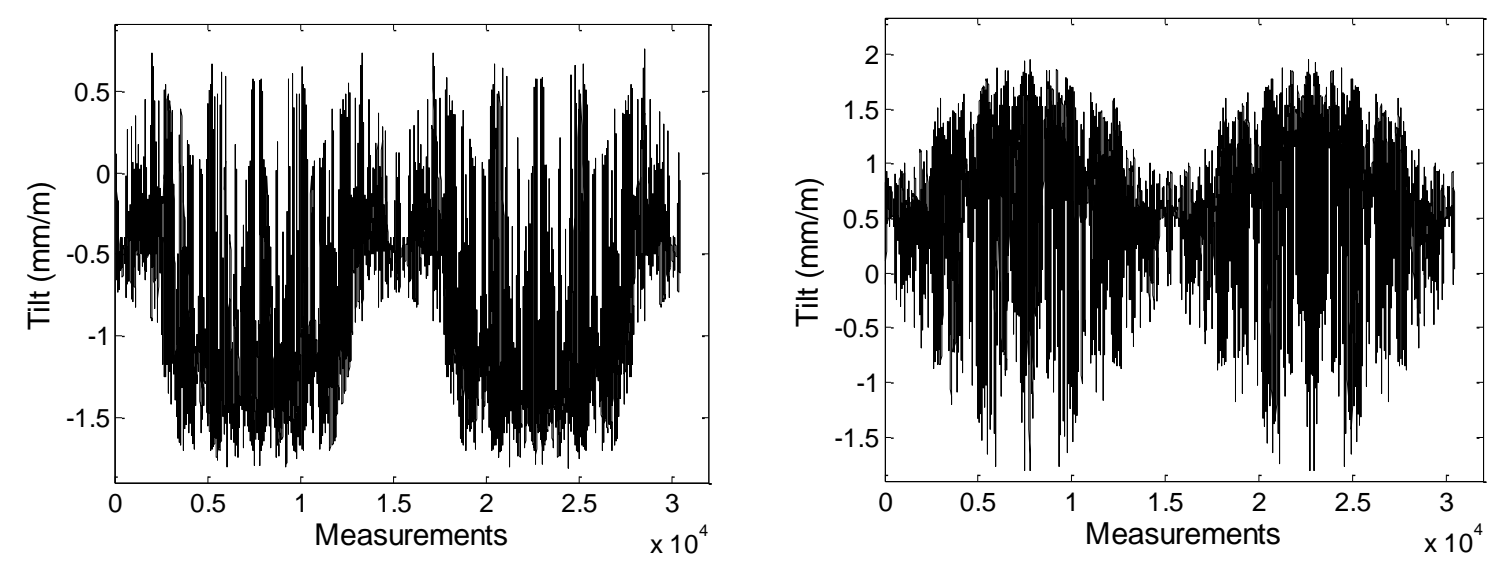

Figure 9: Tilt measurements from sensors TL-1 (left) and TL-4 (right) on the NPL footbridge
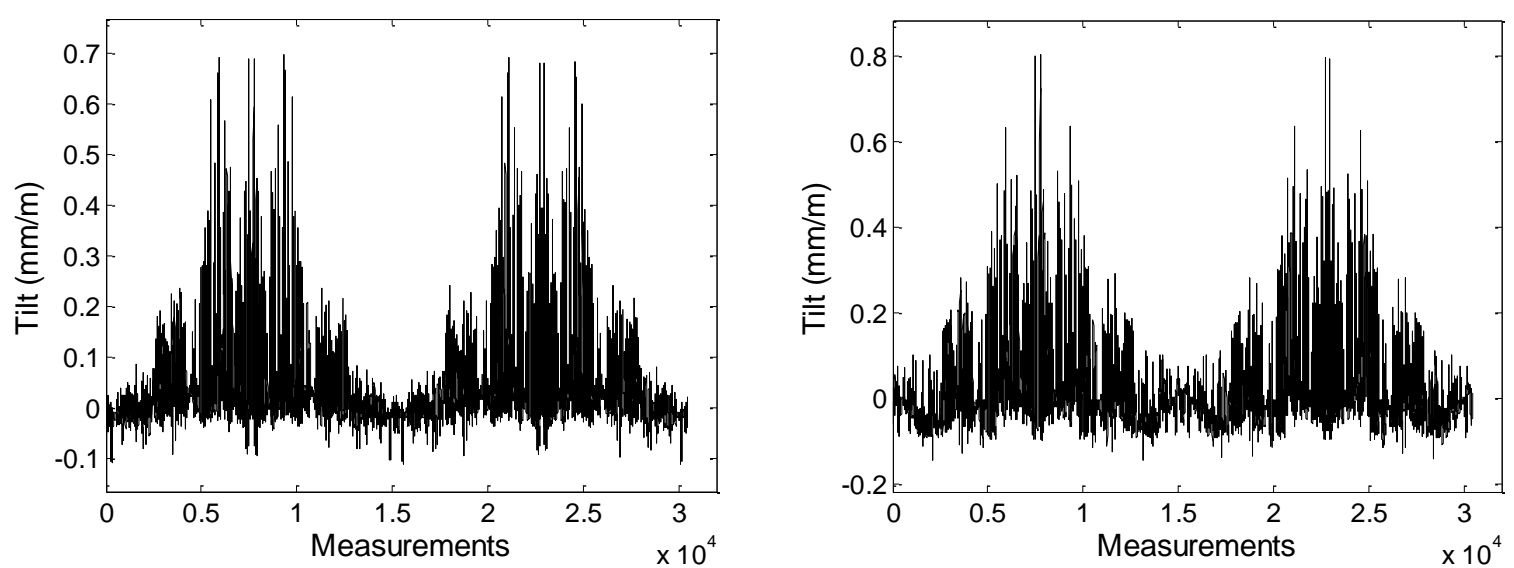

Figure 10: Tilt measurements from sensors TL-2 (left) and TL-3 (right) on the NPL footbridge

Table 4: Maximum and minimum tilt measurements from the NPL footbridge

\begin{tabular}{lcccc}
\hline & TL-1 & TL-2 & TL-3 & TL-4 \\
\hline Minimum tilt $(\mathrm{mm} / \mathrm{m})$ & 0.76 & 0.70 & 0.80 & 1.94 \\
Maximum tilt $(\mathrm{mm} / \mathrm{m})$ & -1.81 & -0.12 & -0.15 & -1.81 \\
\hline Range $(\mathrm{mm} / \mathrm{m})$ & 2.57 & 0.82 & 0.95 & 3.75 \\
\hline
\end{tabular}

Seasonal variations in ambient temperatures are discernible in Figure 11 where the time-series of temperatures collected by sensor TEMP- 6 are plotted. The temperature distribution across a structure is also dependent on the local environmental conditions. One side of the footbridge is closer to nearby trees (see Figure 7) and is hence relatively less exposed to the sun. This aspect results in one side of the bridge experiencing much higher temperatures than the other. This is evident from Table 5, which lists the maximum and minimum temperatures measured by the various sensors over the considered monitoring period. Sensors TEMP1, TEMP-3 and TEMP-5, which are in the shade, measure significantly lower maximum temperatures than the others. 


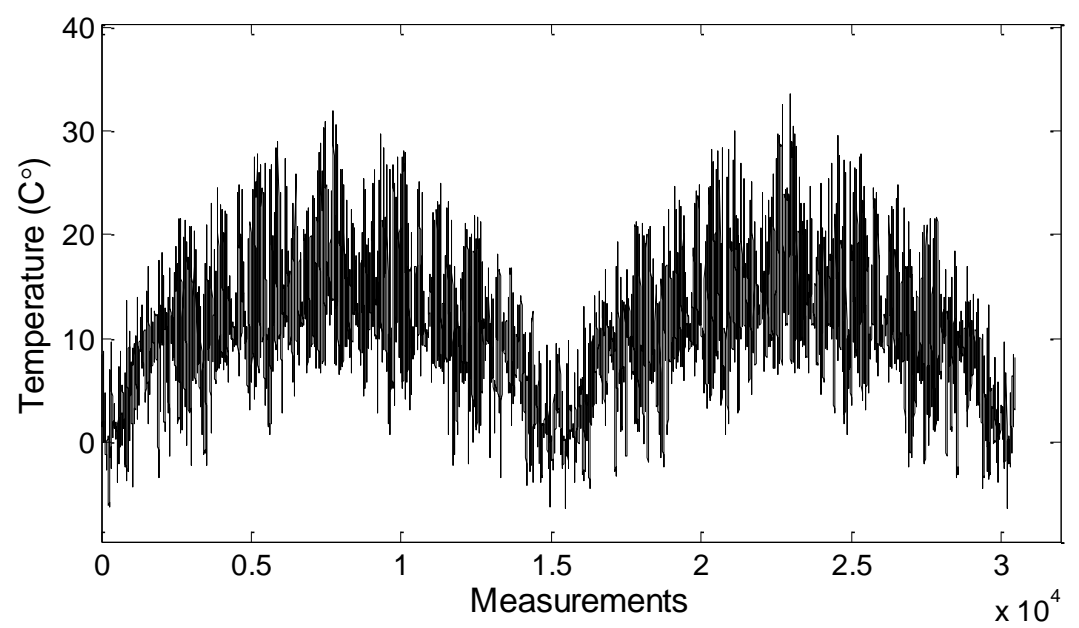

Figure 11: Temperature measurements from sensor TEMP-6 on the NPL footbridge

Table 5: Maximum and minimum temperatures measured by sensors TEMP-1 to TEMP-10 on the NPL footbridge

\begin{tabular}{lccccccccccc}
\hline & \multicolumn{10}{c}{ Temperature sensor (TEMP-i) } \\
\hline$i$ & 1 & 2 & 3 & 4 & 5 & 6 & 7 & 8 & 9 & 10 \\
\hline $\operatorname{Max}\left({ }^{\circ} \mathrm{C}\right)$ & 26.8 & 31.2 & 27.7 & 31.7 & 26.3 & 33.6 & 33.4 & 35.0 & 34.5 & 35.4 \\
$\operatorname{Min}\left({ }^{\circ} \mathrm{C}\right)$ & -6.3 & -6.6 & -6.5 & -6.4 & -6.3 & -6.4 & -6.2 & -6.5 & -6.5 & -6.4 \\
\hline Range $\left({ }^{\circ} \mathrm{C}\right)$ & 33.1 & 37.8 & 34.2 & 38.1 & 32.6 & 40.0 & 39.6 & 41.4 & 41.0 & 41.8 \\
\hline
\end{tabular}

\section{Data pre-processing}

\subsection{Outliers and noise}

Data pre-processing is an essential first step in the measurement interpretation process. In this study, raw data sets are pre-processed to handle outliers and noise [36]. The resulting data is subsequently used to generate regression models. The pre-processing step is essential to generate regression models with high prediction accuracy. The data pre-processing phase is illustrated in Figure 3. In the first stage of this phase, outliers are identified and replaced with appropriate values. This research employs moving windows of specified sizes to determine outliers. Inter-quartile range (IQR) analysis is used to classify if the value at the centre of a moving window is an outlier by comparing it to other values within that window. If the value is classified as an outlier, it is replaced by the median value of the moving window [14, 25]. Noise in measurements is then reduced by smoothing the data using moving averages [37].

Measurements of both the laboratory truss and the NPL footbridge are pre-processed using IQR analysis and smoothing with moving windows of 12 measurements. This has been found to replace potential outliers and average noisy data more efficiently than smaller or larger sized windows. Examples of raw and pre-processed strain and temperature measurements from the laboratory truss are given in Figure 12. Strain recordings are often significantly noisier than temperatures and hence smoothing strain measurements is particularly important for generating accurate regression models. 

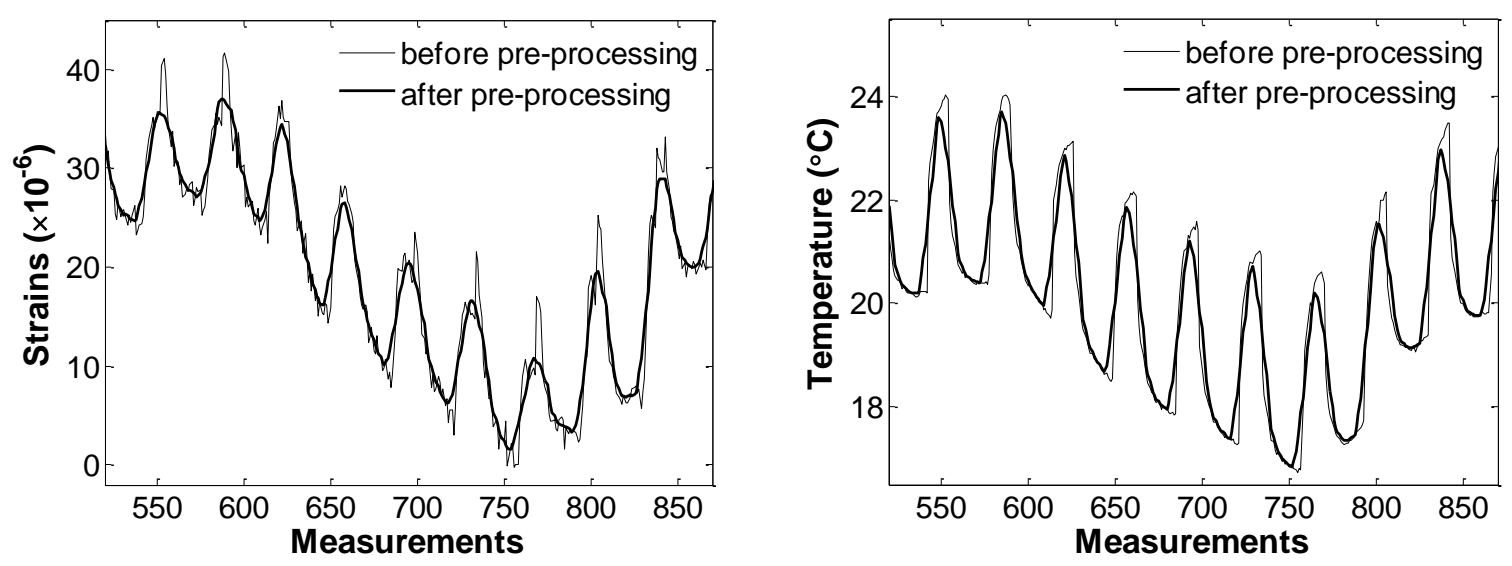

Figure 12: Strain and temperature measurements from sensors S-2 (left) and TEMP-3 (right) on the laboratory truss before and after outlier pre-processing.

\subsection{Training and test sets}

The testing and training sets are generated differently for the two structures due to the difference in the quality and quantity of measurements collected from them. The size of the data set from the laboratory structure is considerably smaller than the one from the NPL footbridge. The truss is kept indoors and therefore not exposed to ambient effects such as sunlight, rain and wind. The seasonal temperature effects are also minimal since measurements from only a 16-day period are used in this study. For this structure, measurements are equally divided into testing and training sets. Measurements taken over the first 7 days, which make up a total of 2000 data-points, constitute the training set. The remaining 2590 measurements are used to evaluate the performance of the proposed algorithms.

The measurement histories from the NPL footbridge show strong seasonal trends since the structure is monitored for a longer period and is also exposed to the outside environment. One year of measurements is required to train adequate regression models. This data-set is hence split into two equal halves: the first half, which is composed of 15,244 time-points and corresponds to duration of almost one year, forms the training set and the second half is used to validate the proposed methodology.

\subsection{Dimensionality reduction}

Reducing the dimensionality of data-sets can help speed up the model generation process and also lead to robust regression models. Principal component analysis (PCA) [38] is a widelyemployed statistical technique that takes advantage of inherent correlations between variables in the data-set for dimensionality reduction. It first involves finding a set of principal component (PC) vectors that define an orthogonal transformation from the original set of linearly-correlated variables to a new set of uncorrelated variables. Then, a small number of $\mathrm{PC}$ vectors, which is known to sufficiently capture the variance in the original data, is chosen to transform the raw data to a low-dimensional PC space.

In this research, PC vectors are first estimated from measurements taken from all temperature sensors over the training period and then used to transform newly collected temperatures to PC space. The transformed data is then given as input to the regression models for thermal response prediction. Dimensionality reduction can, however, negatively impact the accuracy 
of the regression models. Therefore, this research investigates the relationship between the number of chosen PC vectors and the performance of the generated regression models.

The general equation describing PCA is as follows:

$$
D=X P^{T}+M
$$

$D, X, P$ and $M$ are matrices where $D$ stands for the original set of data-points, $X$ for the scores, i.e., the equivalent values in PC space, $P$ for the set of principal components and $M$ for the mean values of the variables. As applied to this work, $D$ of size $m \times n$ represents the timeseries of temperature measurements with $m$ and $n$ denoting the number of temperature sensors and measurements respectively in a moving window; $X$ of size $n \times c$ denotes the matrix of scores with $c$ indicating the number of PCs chosen. After computing the PC vectors from the training set, subsequent measurements of real-time temperatures collected at any time-step $i$ are transformed to PC space using the following equation:

$$
X_{i}=\left(D_{i}-M\right) P
$$

\section{Results}

\subsection{Predicted response}

The various algorithms for generating regression models such as MLR, RR, ANN and SVR are compared in terms of the average prediction error $\left(e_{y}\right)$, which is derived from the predicted $\left(y_{p}\right)$ and measured $\left(y_{r}\right)$ response using the following equation:

$$
e_{y}=\frac{1}{n} \sum_{i=1}^{n}\left|y_{p i}-y_{r i}\right|
$$

$n$ is the number of measurements in the test set and $i$ is the measurement time-step.

\subsubsection{Laboratory structure}

The RBTRP methodology is first evaluated on measurement sets from the laboratory structure. Dimensionality reduction is performed on both raw data-sets and data-sets that have been pre-processed for outliers and noise. Models for thermal response prediction are generated using all discussed regression algorithms. Figures 13 and 14 show the relationship between average prediction error and the number of PCs used as input to the regression models for all strain sensors and for all regression algorithms. For the purposes of illustrating model performance, the predictions from a SVR model using all PCs for strain sensor S-3 are plotted against measured response in Figure 15. The figure shows that model predictions follow measured response very closely.

In figures 14 and 15, the values for the number of PCs that lead to minimum errors are circled for each sensor in the figures. MLR, SVR and RR lead to reliable models as evidenced by the small values for the average prediction error. For these regression algorithms, the largest errors occur when only the first PC is used. The prediction error then generally decreases with increasing number of PCs although this relationship is not monotonic. This can be attributed to not all temperature measurements being strongly correlated to response measurements. Identifying individual temperature measurements that determine the response at a specific location and using only these as input to regression models can help overcome this weakness. However, this aspect is not studied in this paper. The prediction errors for the strain sensors, which are located on the bottom chord (S-1, S-2 and S-3) of the truss, stabilize when two or 
more PCs are used; however, the prediction error for S-4 stabilizes only when 4 or more PCs are used.

In contrast to other regression algorithms, the performance of ANNs varies significantly with the number of PCs employed for model training; the prediction error does not follow a definitive trend with increasing numbers of PCs as seen in Figure 14.
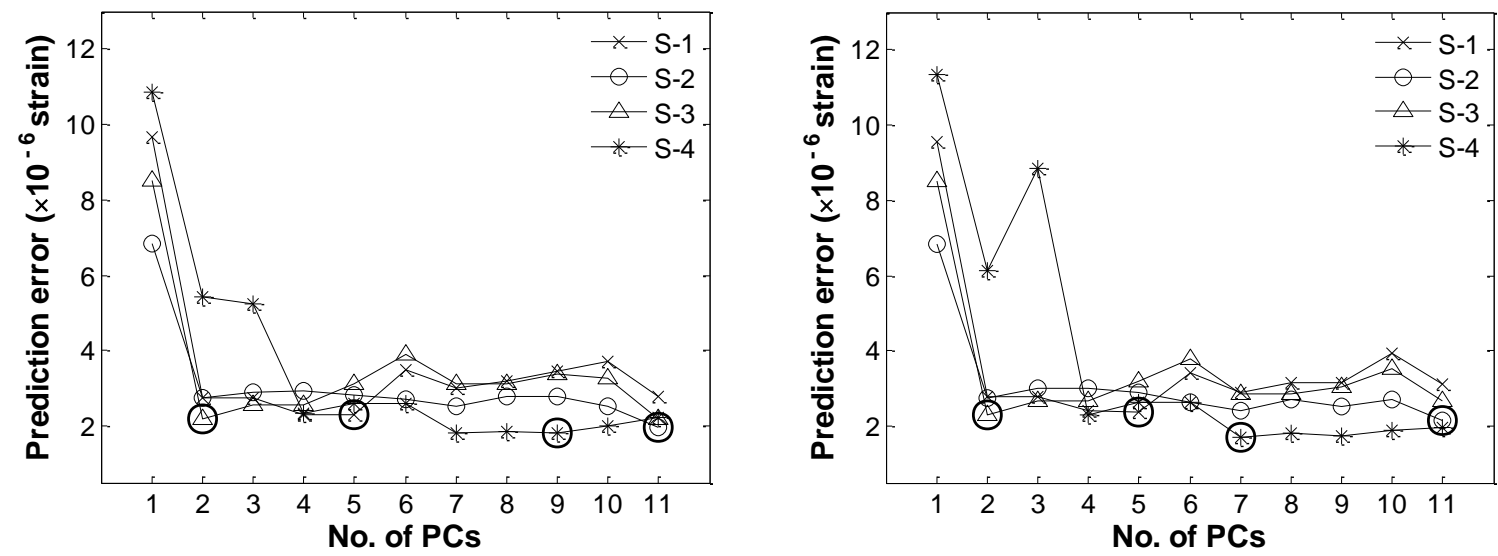

Figure 13 Average strain error from post-processed data sets: MLR (left) and RR (right). Circled elements indicate the minimum average strain error.
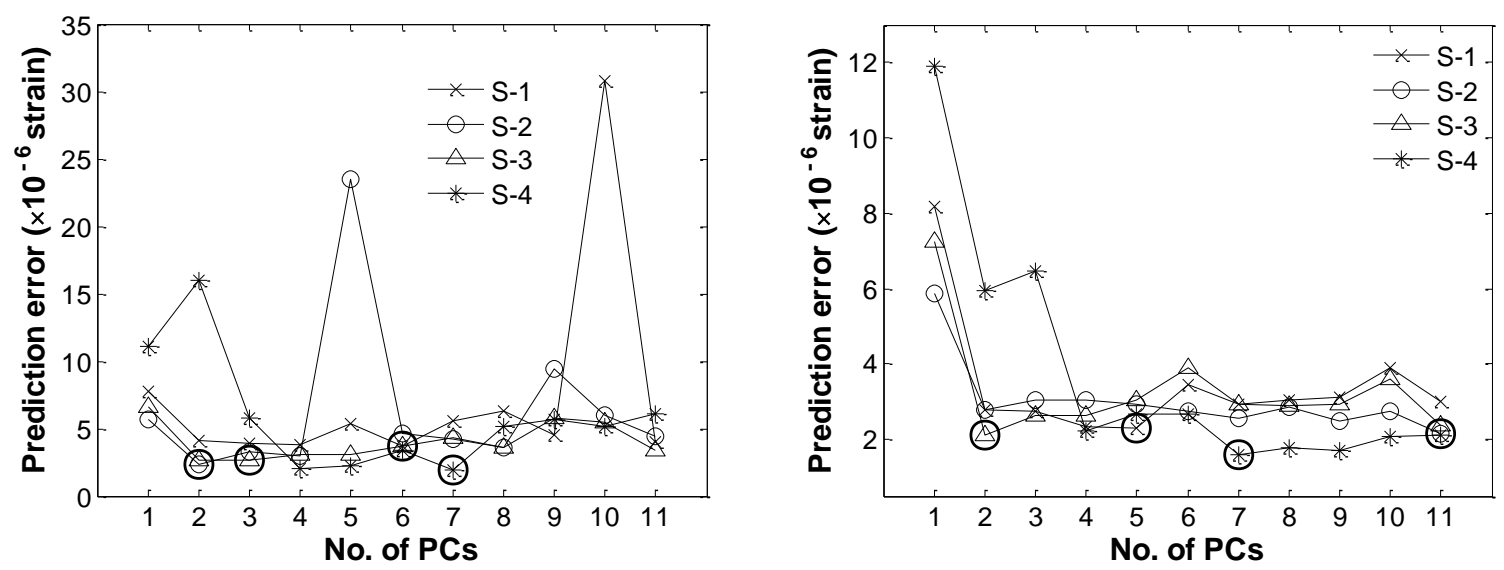

Figure 14: Average strain error from pre-processed data sets: ANN (left) and SVR (right). Circled elements indicate the minimum average strain error.

Results in Figures 13 and 14 show that the decrease in prediction error is insignificant beyond four PCs. Therefore reducing the dimensionality of data-sets enables using less computational time for model training while maintaining high prediction accuracy. This is particularly useful for structures with large numbers of sensors where the training of models could become time and resource-intensive if measurements from all the sensors are used.

The minimum average prediction errors obtained for the models generated by the various regression algorithms are summarized in Table 6. Data in the table show that pre-processing the data for outliers and noise has not significantly altered prediction accuracy. However, this is due to the laboratory conditions not inducing significant noise or outliers in the measurements. Results also show that all algorithms are capable of generating accurate and robust regression models as indicated by the low values for the mean and standard deviation of the prediction error. 


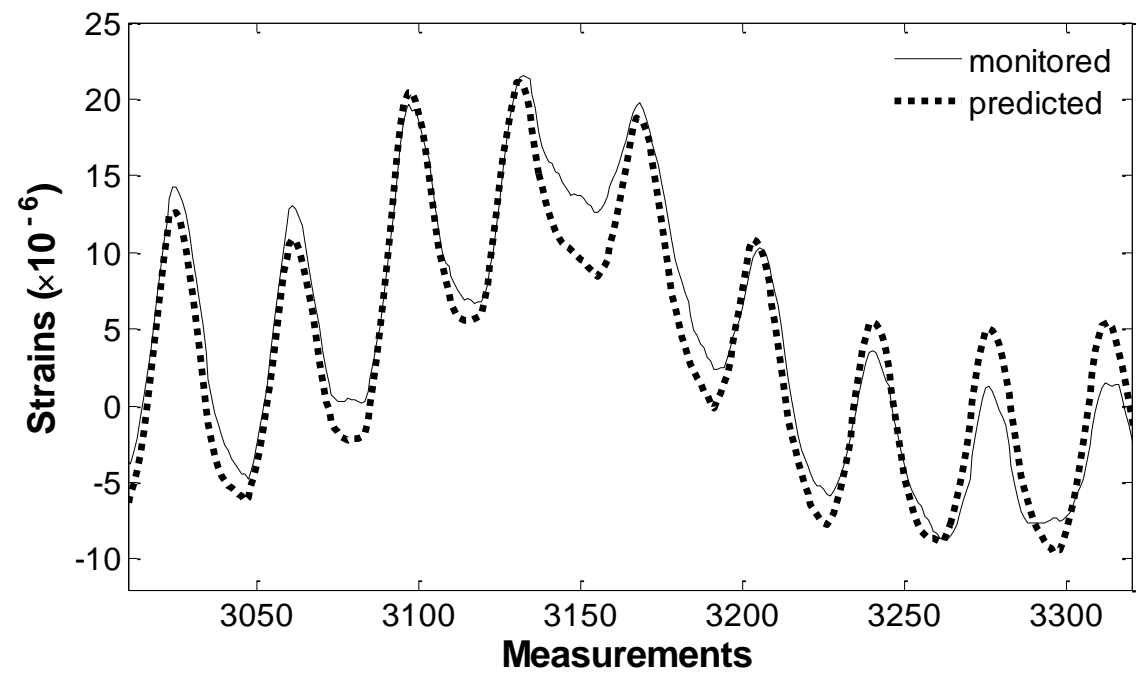

Figure 15: Predictions from a SVR model giving the response at sensor S-2 on the laboratory truss and corresponding measured strains

Table 6: Prediction error $\left(\times 10^{-6}\right.$ strain) of regression models for data-sets from the laboratory truss

\begin{tabular}{lcccccc}
\hline Algorithm & S-1 & S-2 & S-3 & S-4 & Mean & Standard deviation \\
\hline MLR & 2.30 & $\mathbf{1 . 9 8}$ & 2.22 & 1.83 & 2.08 & $\mathbf{0 . 2 2}$ \\
MLR* & $\mathbf{2 . 2 4}$ & 2.00 & 2.13 & 1.66 & $\mathbf{2 . 0 0}$ & 0.25 \\
RR & 2.37 & 2.16 & 2.31 & 1.71 & 2.14 & 0.30 \\
RR* & 2.29 & 2.16 & 2.19 & $\mathbf{1 . 5 4}$ & 2.05 & 0.35 \\
ANN & 3.17 & 2.52 & 2.16 & 2.66 & 2.63 & 0.42 \\
ANN* & 3.66 & 2.36 & 2.62 & 1.93 & 2.64 & 0.74 \\
SVR & 2.37 & 2.06 & 2.27 & 1.81 & 2.13 & 0.25 \\
SVR* & 2.30 & 2.14 & $\mathbf{2 . 1 2}$ & 1.59 & 2.04 & 0.31 \\
\hline *pre-processed for outliers and noise & & & &
\end{tabular}

\subsubsection{The NPL footbridge}

The data-sets collected from the NPL footbridge have a large number of samples due to the high frequency of measurement collection. The time and resource requirements for generating regression models, especially with algorithms of high levels of complexity (e.g., SVR, ANN), increase significantly with the size of data-sets. This research therefore explores if reducing the size of the training data-sets by reducing the frequency of measurement collection or, in this case, simply ignoring measurements would speed up model generation with little loss in prediction accuracy. The actual frequency of measurement collection $(f)$ in the data-set is 3 measurements per hour. In this study, the frequency is artificially varied from 3 measurements per hour to 1 measurement every 3 hours by regularly omitting measurements in the original data-set. For this purpose, regression models are first generated for various sizes of the training data-set and the performance of the models are then studied in terms of the average prediction errors. While the size of the training sets are changed, the data-sets still present the full variability in the measurements as only the number of input measurements is modified; the duration of measurement collection is left unchanged.

The NPL footbridge, which is a concrete bridge, behaves differently to the previously-studied aluminium truss, which due to its material properties immediately responds to changes in ambient temperature. Concrete structures are more voluminous than metal structures; hence 
material temperature varies not only along the surface but also internally across the depth of the sections of elements [39]. Due to its high thermal mass and low thermal conductivity, internal temperatures within concrete elements may lag significantly behind the ambient temperature. This phenomenon is often referred to as thermal inertia. Hua et al. [9] proposed a "dynamic" approach to capture the effects of thermal inertia in their research investigating the relationship between vibration modes and ambient temperatures. A similar approach is adopted in this research. The input to the regression models is composed of both current $\left(D_{i}\right)$ and former temperature $\left(D_{i-j}\right)$ measurements that have been transformed into PC space, i.e. $\left\{\begin{array}{ll}X_{i} & X_{i-j}\end{array}\right\} ; i$ refers to the most recent measurement and $j$ to a previous measurement set collected $j$ time-steps before $i$. From here onwards, $j$ is referred to as the thermal inertia parameter.

This sub-section therefore presents results from investigations on the influence of the following features on prediction accuracy for regression algorithms:

(i) number of selected PCs;

(ii) number of input measurement $\left(f_{i}\right)$; and

(iii) thermal inertia parameter $(j)$.

Figure 16 illustrates the variation of average prediction errors from regression models generated using SVR for tilt sensors TL-1 and TL-2; prediction errors are plotted against the number of PCs for different numbers of input measurements. Data-sets are pre-processed for outliers and noise, and the thermal inertia parameter, $j$ is equal to 1 . In most cases, the prediction error reduces as the number of inputs, i.e., the number of PCs, is increased. The plots in Figure 16 show that the number of measurement inputs employed to train the regression algorithm directly affects the prediction accuracy. While the prediction error reduces with increasing frequency of measurement collection, the improvement in prediction error is negligible when a sufficient number of PCs are used. For tilt sensors TL-1 and TL-2, the prediction errors are small if 3 PCs and a measurement frequency of 1 measurement every 3 hours are specified (see Figure 16).

Next, the study analyses the influence of the thermal inertia parameter $j$ on model performance. Corresponding results, which are illustrated in Figure 17 and 18, show that there is an optimal value for $j$ for which the models have minimum prediction errors. In figure 17, the variation of prediction errors with thermal inertia parameter $j$ for tilt sensor TL- 1 has a sinusoidal pattern. Prediction errors reach their minimum when $j=36$ and $j=108$. The periodicity of the pattern is therefore approximately 72 measurements, which is equivalent to a time interval of one day. Figure 18 shows the variation of prediction errors with number of PCs and the thermal inertia parameter $j$ for each sensor. $j$ is varied from 1 to 40 ; the prediction error is minimum for $j=36$. These results illustrate that the thermal response at any timeinstant is determined by the current set of temperature measurements and those collected 36 time-steps earlier. For the NPL footbridge, $j=36$ corresponds to a time interval of half a day; this value therefore suggests thermal lag in the structure caused by internal temperatures that are closer to the ambient temperature taken 12 hours earlier in the day. Furthermore, the plots also show that the thermal inertia parameter has a larger impact on the prediction errors for sensors TL-1 and TL-4 than for TL-2 and TL-3. The reason for such behaviour may be due to TL-1 and TL-4 being on the overhanging portions of the footbridge, which are much less constrained to deform under thermal effects than its main-span. 

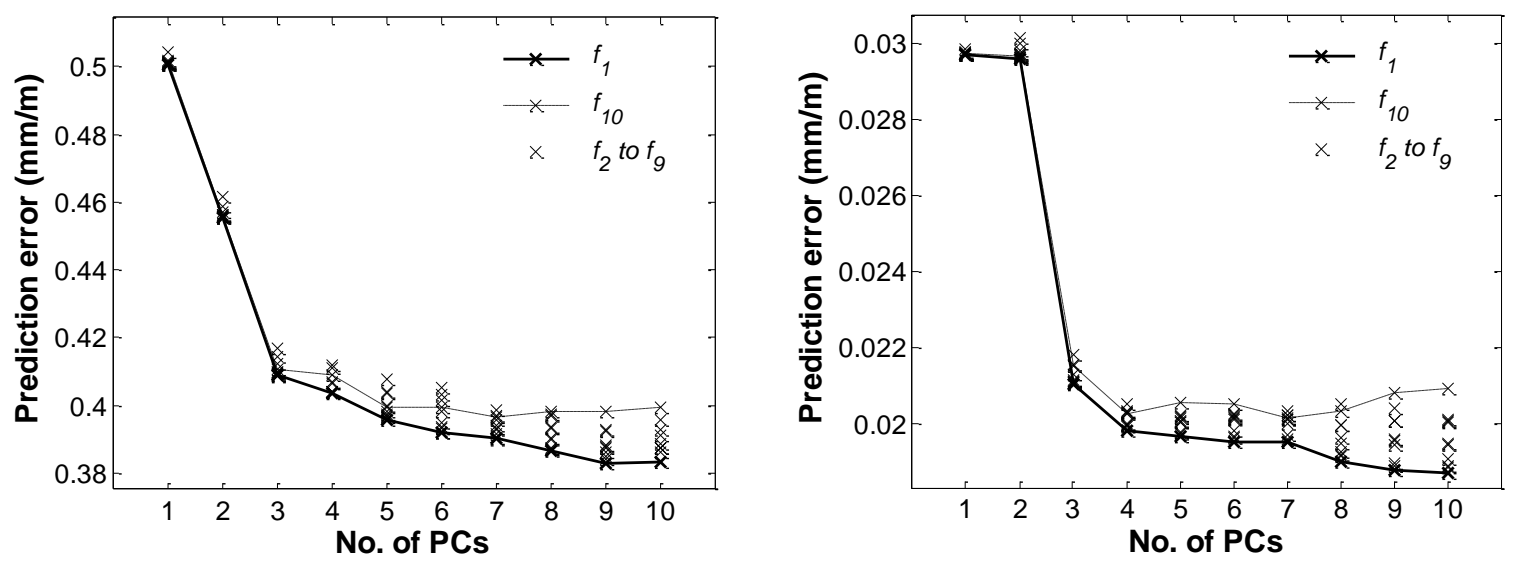

Figure 16: Tilt prediction errors ( $\mathrm{mm} / \mathrm{m}$ ) using SVR models for sensors TL-1 (left) and TL-2 (right).

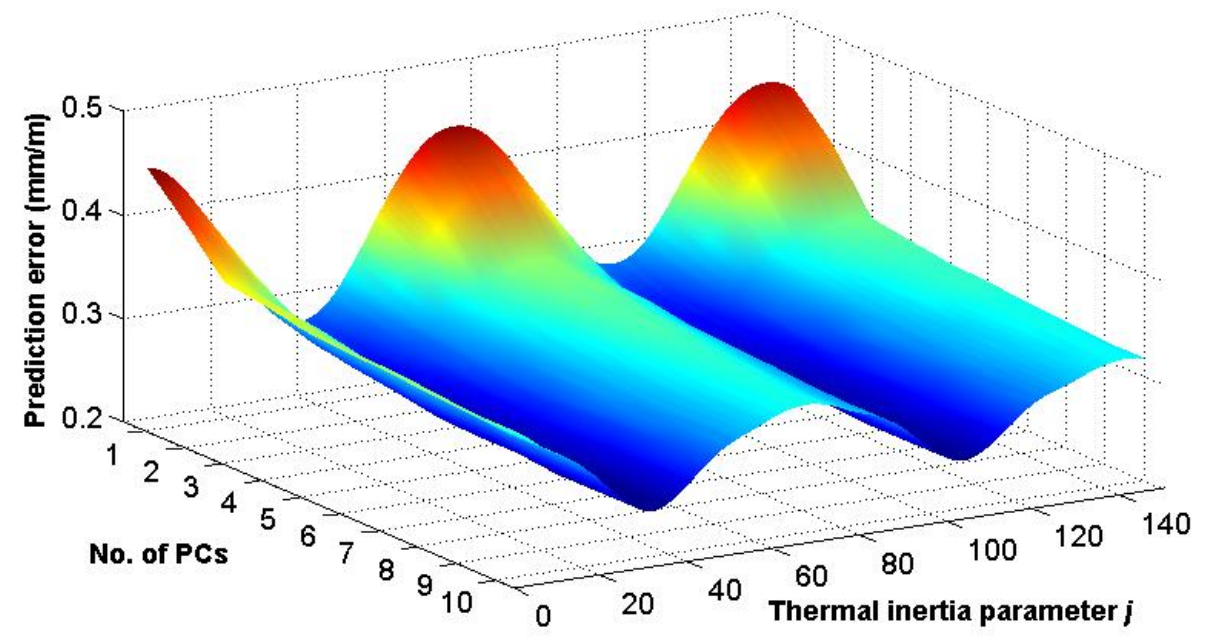

Figure 17: Tilt prediction error $(\mathrm{mm} / \mathrm{m})$ versus number of PCs and thermal inertia parameter $j$ from sensor TL-1

The minimum tilt errors generated by the various regression algorithms for all tilt sensors are given in Table 7. Results in the table show that pre-processing the measurements to manage outliers and noise reduces prediction error by as much as $21 \%$. Of the regression algorithms studied, ANNs provide the minimum prediction errors for the NPL footbridge. Comparing these results to those from the laboratory truss, we can conclude that the choice of the regression algorithms used to model the temperature-response relationship is dependent on the structure. The NPL footbridge is a concrete bridge. Changes in ambient temperature or solar radiation are not immediately reflected in its response due to thermal inertia effects arising from its low thermal conductivity and high thermal mass. The laboratory truss is made of aluminium, a material with superior thermal conductivity, and therefore minimal thermal inertia effects Furthermore, mechanical properties of concrete such as its elastic modulus are also known to vary considerably with changes in temperature in comparison to aluminium. Consequently, the nature of the relationship between temperatures and structural response for the NPL footbridge and the laboratory truss are likely to be very different. Generalizing this, the choice of regression model would be dependent on the structural system in consideration. This is also in keeping with the well-known no-free-lunch theorem [40], which states that there is no single algorithm that is optimal for all problem classes. 
As noted in Table 7, the prediction errors observed for tilt sensors TL-2 and TL-3 are significantly less than those for tilt sensors TL-1 and TL-4. This may be attributed to the fact that measurements at TL-2 and TL-3 are more highly correlated with temperature measurements than TL-1 and TL-4. However, the prediction errors are still not large in magnitude when compared in terms of the range of measurements collected at these sensors (see Table 4). TL-1 and TL-4 measure much larger tilts than TL-2 and TL-3 and therefore, the normalized values of the errors are similar in magnitude. To illustrate this aspect, model predictions and measurements at tilt sensors TL- 1 and TL-2 are compared in Figures 19 and 20 respectively. For both sensors, model predictions closely follow actual measurements.
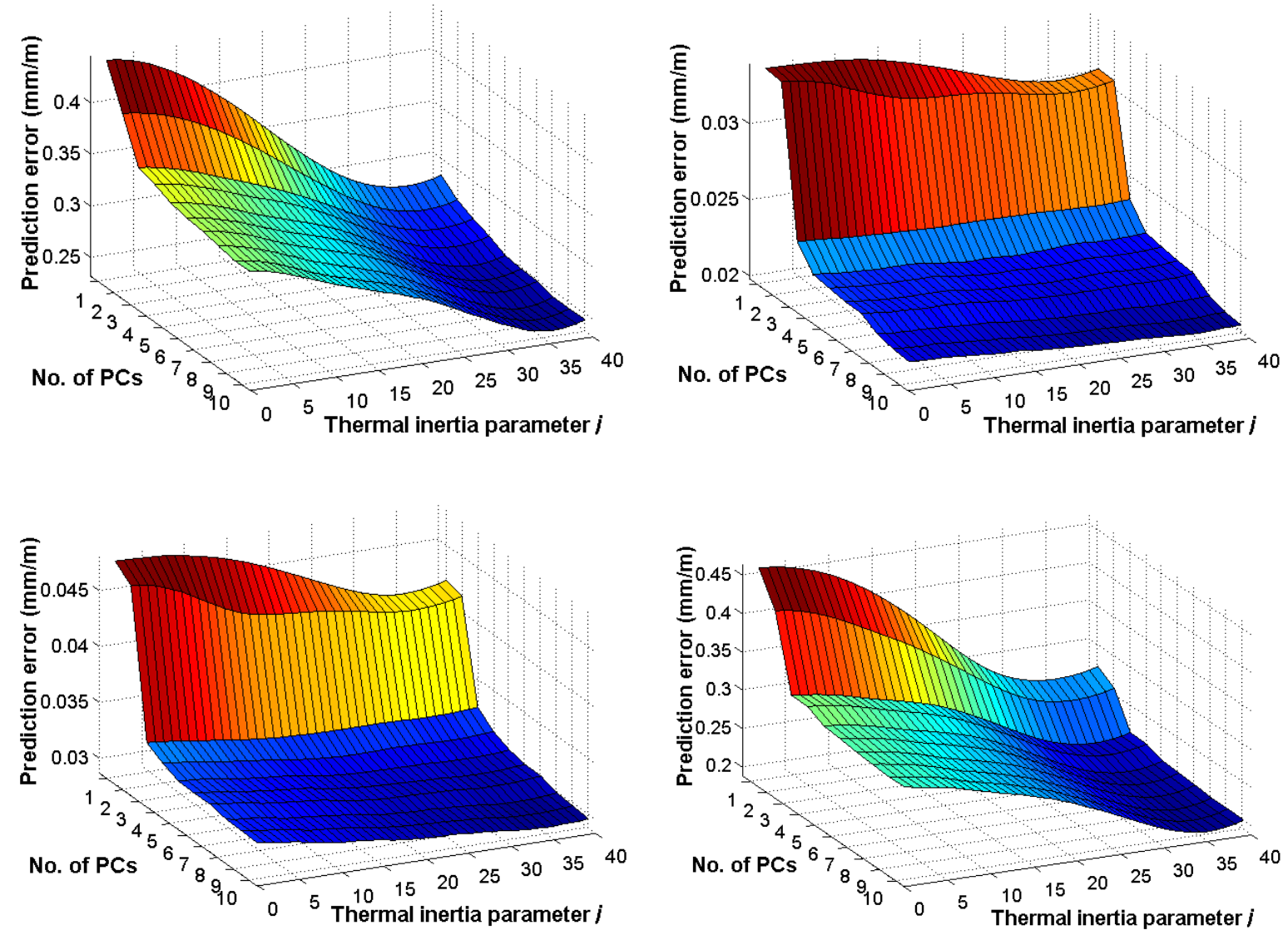

Figure 18: Tilt prediction error $(\mathrm{mm} / \mathrm{m})$ versus number of PCs and thermal inertia parameter $j$ from sensor TL-1 (top left), TL-2 (top right), TL-3 (bottom left) and TL-4 (bottom right).

Table 7: Average tilt error in $\mathrm{mm} / \mathrm{m}$

\begin{tabular}{lcccccc}
\hline Algorithm & TL-1 & TL-2 & TL-3 & TL-4 & Mean & Standard deviation \\
\hline MLR & 0.273 & 0.026 & 0.036 & 0.236 & 0.143 & 0.130 \\
MLR* & 0.242 & 0.021 & 0.030 & 0.194 & 0.122 & 0.113 \\
RR & 0.271 & 0.026 & 0.036 & 0.235 & 0.142 & 0.129 \\
RR* & 0.240 & 0.020 & 0.029 & 0.193 & 0.121 & 0.112 \\
ANN & 0.225 & 0.022 & 0.030 & 0.195 & 0.118 & 0.107 \\
ANN* & $\mathbf{0 . 1 8 2}$ & $\mathbf{0 . 0 1 6}$ & $\mathbf{0 . 0 2 3}$ & $\mathbf{0 . 1 4 6}$ & $\mathbf{0 . 0 9 2}$ & $\mathbf{0 . 0 8 5}$ \\
SVR & 0.274 & 0.026 & 0.037 & 0.242 & 0.145 & 0.131 \\
SVR* & 0.237 & 0.020 & 0.029 & 0.192 & 0.120 & 0.111 \\
\hline *pre-processed & & & & & \\
\hline
\end{tabular}




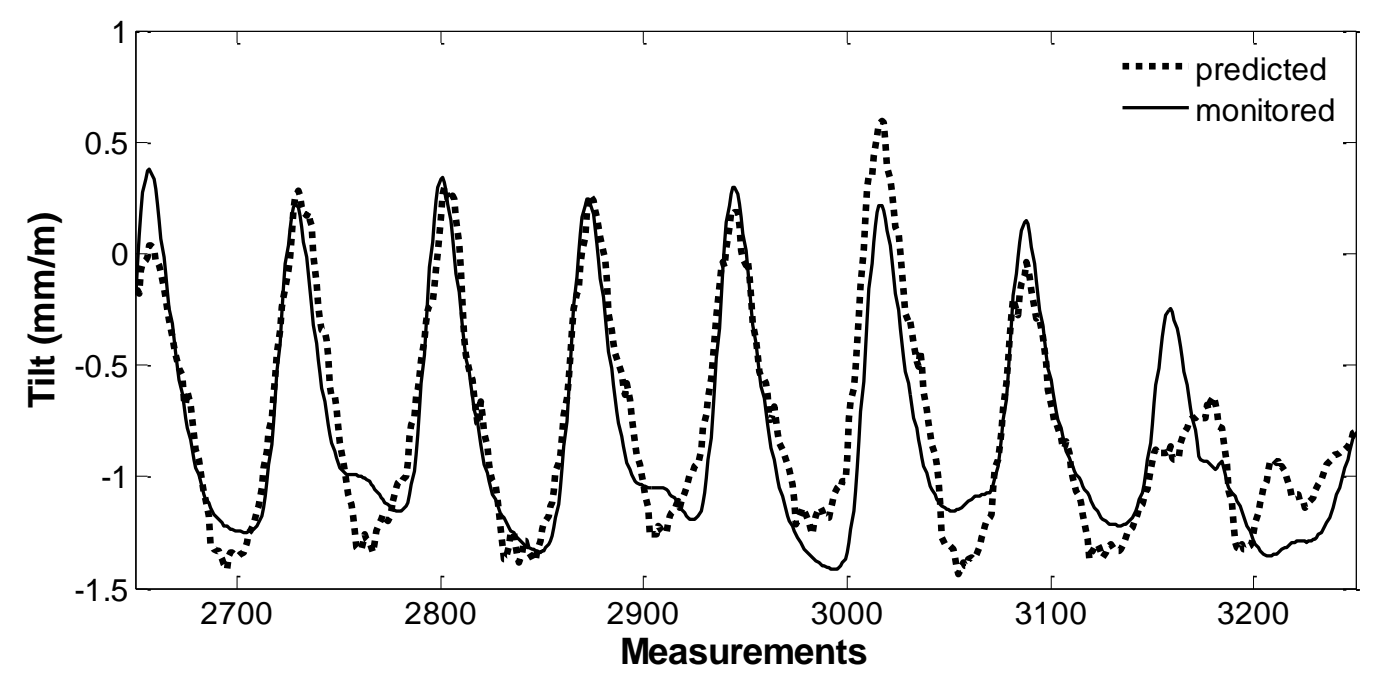

Figure 19: Predictions from a SVR model for tilt sensor TL-1 on the NPL footbridge and corresponding measurements over a 9-day period.

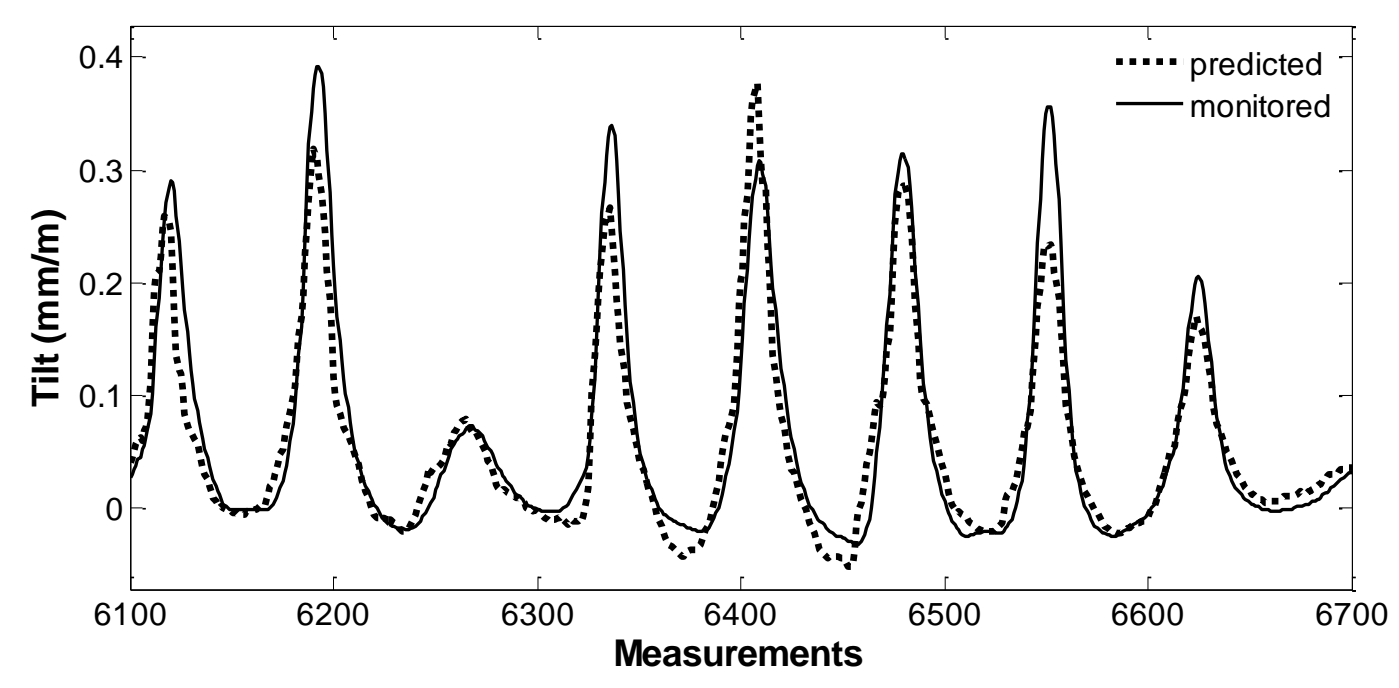

Figure 20: Predictions from a SVR model for tilt sensor TL-2 on the NPL footbridge and corresponding measurements over a 9-day period.

\section{Summary and Conclusions}

This paper proposes a data-driven strategy, referred to as Regression-Based Thermal Response Prediction (RBTRP) methodology, for predicting thermal response of structures from distributed temperature measurements. The main idea of the methodology is to develop statistical regression models, which sufficiently capture the physical relationships between structural response and temperature distributions, from distributed temperature and response measurements collected during a reference period. This study investigates the application of various statistical techniques such as MLR, RR, ANN and SVR for the generation of regression models and then evaluates their performance on measurements taken from two structures - a laboratory truss and a full-scale footbridge at the NPL.

This study has led to the following findings: 
- The proposed RBTRP methodology leads to regression models that accurately predict the thermal response of bridges from distributed temperature measurements.

- All of the studied regression algorithms integrate effectively within the RBTRP methodology. However, the optimal algorithm for a specific monitoring project is determined by factors such as its structural properties and the quality of collected measurements.

- The number of samples employed for training regression models can be reduced without greatly affecting the prediction accuracy. However the training data-set must encompass the full range of variability in measurements. For a full-scale structure, this implies that measurements covering at least one year may be required for training.

- Using principal component analysis (PCA) to reduce the dimensionality of data-sets given as input to the regression models does not significantly compromise prediction accuracy.

- Effects of thermal inertia are effectively modelled within the proposed methodology. As seen from results for the NPL footbridge, this can be accomplished by providing both current as well as previous temperature measurements as inputs to regression models.

Future work will investigate embedding the proposed RBTRP methodology within an anomaly detection strategy that examines the difference between thermal response predictions and real-time measurements. Research will examine ways to cluster sensors prior to model training to identify individual temperature measurements that are most relevant for predicting response at specific locations. Thermal inertia effects will be studied in more depth to evaluate if temperatures collected at several time-steps may be required to predict structural response.

\section{Acknowledgements}

The authors would also like to thank Dr Elena Barton and the NPL for making measurements and relevant information on the NPL footbridge available to this research. They would also like to express their gratitude to Bill Harvey Associates and Pembrokeshire County Council for providing access to the data from the Cleddau Bridge.

\section{References}

[1] Ni YQ, Zhou HF, Chan KC, Ko JM. Modal flexibility analysis of cable-stayed ting kau bridge for damage identification. Computer-Aided Civil and Infrastructure Engineering. 2008;23:223-36.

[2] Inaudi D, Bolster M, Deblois R, French C, Phipps A, Sebasky J, et al. Structural health monitoring system for the new i-35w st anthony falls bridge. Proceedings of 4th International Conference on Structural Health Monitoring on Intelligent Infrastructure (SHMII-4). Zurich, Switzerland; 2009.

[3] Brownjohn J. Structural health monitoring of civil infrastructure. Philos Transact A Math Phys Eng Sci. . 2007;365:589-622.

[4] Aktan AE, Tsikos CJ, Catbas FN, Grimmelsman K, Barrish R. Challenges and opportunities in bridge health monitoring. Proceedings of the 2nd International Workshop on Structural Health Monitoring. Stanford, CA, USA, 1999.

[5] Bennet PJ, Stoianov I, Fidler P, Maksimovic C, Middletion C, Graham N, et al. Wireless sensor networks: Creating 'smart infrastructure'. Proceedings of the ICE-Civil engineering: Telford; 2009. p. 136-43. 
[6] Fraser M, Elgamal A, He X, Conte JP. Sensor network for structural health monitoring of a highway bridge. Journal of Computing in Civil Engineering. 2010;24:11-24.

[7] Lynch JP, Loh KJ. A summary review of wireless sensors and sensor networks for structural health monitoring. Shock and Vibration Digest. 2006;38:91-130.

[8] Park G, Rosing T, Todd MD, Farrar CR, Hodgkiss W. Energy harvesting for structural health monitoring sensor networks. Journal of Infrastructure Systems. 2008;14:64-79.

[9] Hua XG, Ni YQ, Ko JM, Wong KY. Modeling of temperature-frequency correlation using combined principal component analysis and support vector regression technique. Journal of Computing in Civil Engineering. 2007;21:122-35.

[10] Catbas FN, Susoy M, Frangopol DM. Structural health monitoring and reliability estimation: Long span truss bridge application with environmental monitoring data Engineering Structures. 2008;30:2347-59.

[11] Cross EJ, Koo KY, Brownjohn JMW, Worden K. Long-term monitoring and data analysis of the tamar bridge. Mechanical Systems and Signal Processing. 2013;35:16-34.

[12] Ni YQ, Hu XG, Fan KQ, and Ko JM. Correlating modal properties with temperature using long-term monitoring data and support vector machine technique. Engineering Structures. 2005;27:P. 1762-73.

[13] Goulet JA, Kripakaran P, Smith IFC. Multimodel structural performance monitoring Journal of Structural Engineering. 2010;136:1309-18.

[14] Posenato D, Kripakaran P, Inaudi D, Smith IFC. Methodologies for model-free data interpretation of civil engineering structures. Computers and Structures. 2010;88:467-82.

[15] Moyo P, Brownjohn J. Detection of anomalous structural behaviour using wavelet analysis. Mechanical Systems and Signal Processing. 2002;16:429-45.

[16] Omenzetter P, Brownjohn JMW. Application of time series analysis for bridge monitoring. Smart Materials and Structures. 2006;15:129.

[17] Laory I, Ali NBH, Trinh TN, Smith IFC. Measurement system configuration for damage identification of continuously monitored structures. Journal of Bridge Engineering. 2012;17:NA.

[18] Potgieter IC, Gamble WL. Nonlinear temperature distributions in bridges at different locations in the united states. PCI Journal. 1989;July-August 1989:80-103.

[19] Li ZX, Chanb THT, Zheng R. Statistical analysis of online strain response and its application in fatigue assessment of a long-span steel bridge Engineering Structures. 2003;25:1731-41.

[20] Mata J, Castro ATd, Costa JSd. Time-frequency analysis for concrete dam safety control: Correlation between the daily variation of structural response and air temperature. Engineering Structures. 2013;48:658-65.

[21] Laory I, Trinh TN, Posenato D, Smith IFC. Combined model-free data-interpretation methodologies for damage detection during continuous monitoring of structures. Journal of Computing in Civil Engineering. 2013.

[22] Mehrjoo M, Khaji N, Moharrami H, Bahreininejad A. Damage detection of truss bridge joints using artificial neural networks. Expert Systems with Applications. 2008;35:1122-31.

[23] Ko J, Sun Z, Ni Y. Multi-stage identification scheme for detecting damage in cablestayed kap shui mun bridge. Engineering Structures. 2002;24:857-68.

[24] Zhang J, Sato T, Iai S. Support vector regression for on-line health monitoring of largescale structures. Structural Safety. 2006;28:392-406.

[25] Kromanis R, Kripakaran P. Support vector regression for anomaly detection from measurement histories. Advanced Engineering Informatics. 2013;27:486-95.

[26] Aiken LS, West SG, Pitts SC. Multiple linear regression. In: Schinka JA, Velicer WF, editors. Handbook of psychology. Volume 2: Research methods in psychology. Hoboken, New Jersey: John Wiley \& Sons, Inc.; 2003. p. 484-507. 
[27] Huber PJ. Robust regression: Asymptotics, conjectures and monte carlo. The Annals of Statistics. 1973;1:799-821.

[28] Basheer IA, Hajmeer M. Artificial neural networks: Fundamentals, computing, design, and application. Journal of Microbiological Methods. 2000;43:3-31.

[29] MATLAB. And statistics toolbox release 2011b. The MathWorks, Inc., Natick, Massachusetts, United States.

[30] Dackermann U. Vibration-based damage identification methods for civil engineering structures using artificial neural networks [Dissertation]. Sydney: University of Technology Sydney; 2010.

[31] Mata J. Interpretation of concrete dam behaviour with artificial neural network and multiple linear regression models. Engineering Structures. 2011;33:903-10.

[32] Katsikeros CE, Labeas G. Development and validation of a strain-based structural health monitoring system. Mechanical Systems and Signal Processing. 2009;23:372-83.

[33] Haykin SS. Neural networks: A comprehensive foundation: Prentice Hall; 1999.

[34] Vapnik VN. The nature of statistical learning theroy. 2nd edition. New York: SpringerVerlag New York Inc.; 1999.

[35] Smola AJ, Schölkopf B. A tutorial on support vector regression. Statistics and Computing. 2004;14:199-222.

[36] Kotsiantis SB, Kanellopoulos D, Pintelas PE. Data preprocessing for supervised learning International Journal of Computers Sciences. 2006;1:111-7.

[37] Ganguli R. Noise and outlier removal from jet engine health signals using weighted fir median hybrid filters. Mechanical Systems and Signal Processing. 2002;16:967-78.

[38] Jolliffe IT. Principal component analysis. 2nd Ed., Springer-Verlag, Inc., New York. 2002.

[39] Branco FA, Mendes PA. Thermal actions for concrete bridge design. Journal of Structural Engineering. 1993;119:2313-31.

[40] Wolpert DH, Macready WG. No free lunch theorems for optimization. Evolutionary Computation, IEEE Transactions on. 1997;1:67-82. 